"This is an Accepted Manuscript of an article published by Macromol. Theory and Simul., mats.201700106, accepted in January 2018; vol 27, no 3, 1700106 (2018)."

\title{
Intelligent Monte Carlo: A new paradigm for inverse polymerization engineering
}

Yousef Mohammadi ${ }^{*}$, Mohammad Reza Saeb², Alexander Penlidis ${ }^{3 *}$, Esmaiel Jabbari ${ }^{4}$, Philippe Zinck $^{5 *}$, Florian J. Stadler ${ }^{6}$, Krzysztof Matyjaszewski $^{7 *}$

1. Petrochemical Research and Technology Company (NPC-rt), National Petrochemical Company (NPC), P.O. Box 14358-84711, Tehran, Iran

2. Department of Resin and Additives, Institute for Color Science and Technology, P.O. Box 16765-654, Tehran, Iran

3. Department of Chemical Engineering, Institute for Polymer Research (IPR), University of Waterloo, Waterloo, Ontario N2L 3G1 Canada

4. Biomimetic Materials and Tissue Engineering Laboratory, Department of Chemical Engineering, University of South Carolina, Columbia, SC, USA

5. Unity of Catalysis and Solid State Chemistry, University of Lille, CNRS, Bât C7, Cité Scientifique, 59652 Villeneuve d'Ascq Cédex, France

6. College of Materials Science and Engineering, Shenzhen Key Laboratory of Polymer Science and Technology, Guangdong Research Center for Interfacial Engineering of Functional Materials, Nanshan District Key Lab for Biopolymers and Safety Evaluation, Shenzhen University, Shenzhen 518060, China

7. Department of Chemistry, Carnegie Mellon University, Pittsburgh, USA

To whom correspondence should be addressed:

Dr. Yousef Mohammadi: mohammadi@npc-rt.ir

Prof. Alexander Penlidis: penlidis@uwaterloo.ca

Prof. Philippe Zinck: philippe.zinck@univ-lille1.fr

Prof. Krzysztof Matyjaszewski: matyjaszewski@cmu.edu 


\section{GRAPHICAL ABSTRACT}

Traditional computational methods simulate the microstructure of polymer chains from input polymerization conditions. In this contribution, we introduce the Intelligent Monte Carlo (IMC) approach able to predict optimal recipe/operating conditions for synthesizing complex copolymer molecules with predefined microstructures as input. Chain shuttling copolymerization is chosen as the first test case.

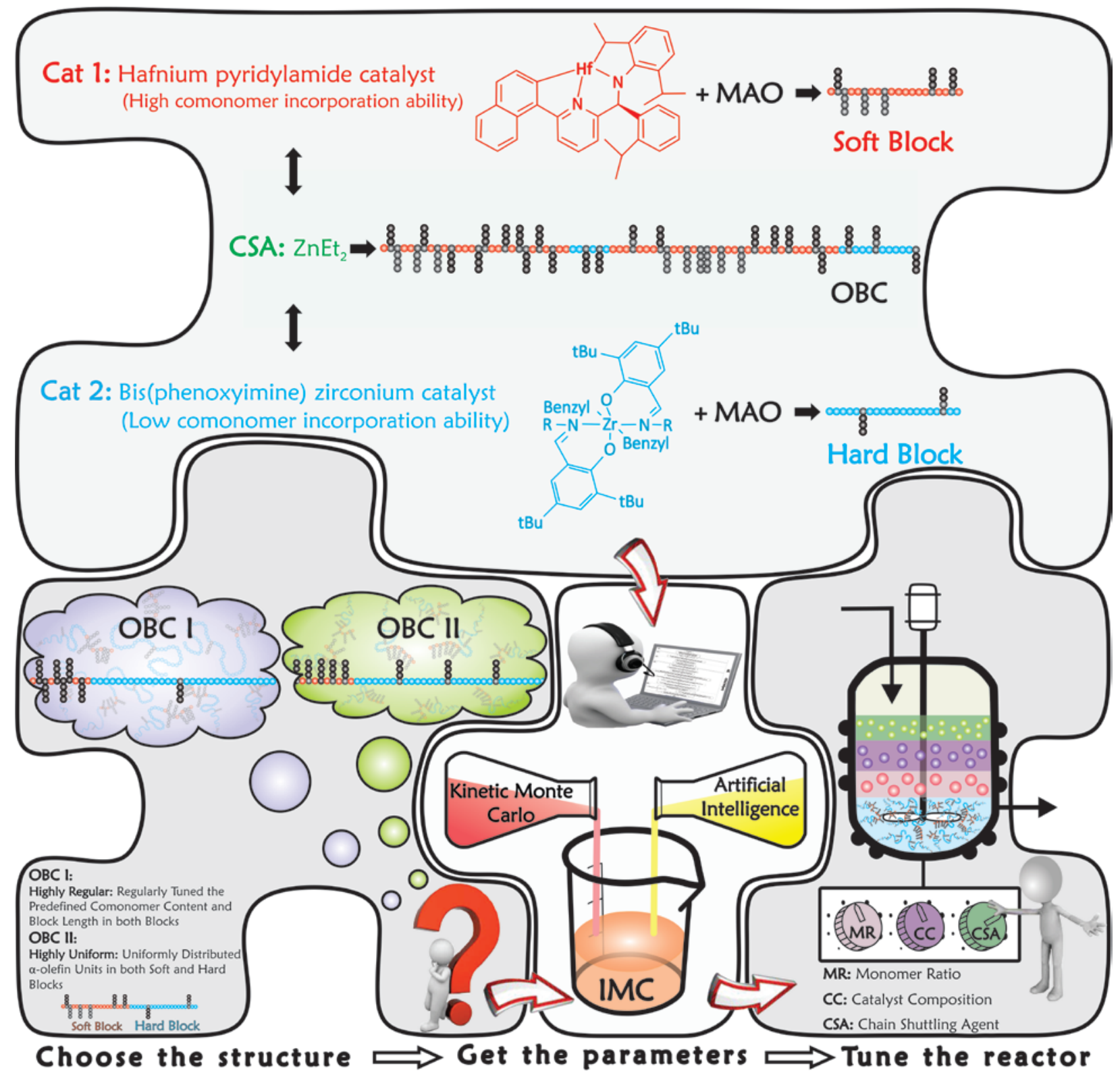




\begin{abstract}
Traditional computational methods simulate the microstructure of polymer chains from input reaction conditions, but a need exists for predicting optimum reaction conditions in a computationally-demanding multi-variable space leading to the synthesis of predesigned microstructures and architectures. We introduce herein the Intelligent Monte Carlo (IMC) approach, able to predict optimum reaction conditions for synthesizing copolymers with predefined, complex microstructures as input. This is rendered possible by a combination of Kinetic Monte Carlo (KMC) simulation with Artificial Intelligence concepts, which enables a reasonably enhanced convergence to optimum reactions conditions. Chain shuttling polymerization was chosen as a first test case due to its complexity and the intricate multi-block microstructures that are formed; whose tailoring requires multiple parameters. The IMC approach located optimum reaction conditions for the synthesis of olefinic multi-block copolymers with specific microstructures. This approach provides a new platform for identifying complex reaction conditions to 'produce' and 'tailor-make' materials with precisely predefined microstructures and facilitates the development of meaningful structure-property relationships.
\end{abstract}

Keywords: Monte Carlo simulation; Chain shuttling polymerization; Artificial Intelligence; Inverse polymerization engineering 


\section{INTRODUCTION}

Over the last decades, computational chemistry has provided a suite of promising tools for all aspects of process and product design and optimization, namely, for studying reaction mechanisms, designing new compounds and materials, and improving chemical processes [1-4]. In many complex systems including industrial processes, numerical simulations are required to predict the outcome of a certain operation performed with a defined set of input variables. Due to the enormous complexity of the nonlinear problem of linking input factors to output properties, it is not possible to predict readily the former from the latter. However, in industrial processes, if one were able to 'dial in' a certain set of resulting characteristics (desirable product properties) and subsequently, via using some modelling/simulation software tool, obtain feasible suggestions for a set of input factors that would give rise to such properties, would be economically highly important and beneficial. In the chemical industry, for example, a significant number of reactions are frequently occurring simultaneously and influence each other.

In the synthetic polymer chemistry, modern monitoring/measuring technology has given rise to increasingly more complex and elaborated reaction mechanisms and product microstructures and architectures, whose description and simulation is not trivial [5-10]. For instance, the sequence (length) distribution of comonomer units along a macromolecular chain is a key property characteristic that governs several macroscopic properties of a polymer. Kinetic Monte Carlo (KMC) simulations have been successfully used to gain detailed micromolecular level information about chain microstructure, as described, for example, in references [11] to [28]. In traditional simulation approaches, the strategy consists of obtaining microstructural signatures of the copolymer chains mainly in response to input reaction conditions. In many practical applications, however, the desired properties of the polymers can be specified and one should ideally be able to move backwards and define the reaction conditions required to obtain the corresponding microstructures. The reverse simulation going from the macromolecular microstructure (as problem input) to the reaction conditions (as problem output) requires searching a large multi-variable space for the optimum conditions for the synthesis of predesigned copolymer microstructures. This (tedious task for deterministic models/simulations) 
can be done by providing typical KMC approaches with the capability to make decisions. KMC should thus be equipped with an appropriate optimization tool to intelligently seek, identify, and screen for molecular patterns in a (potentially huge) copolymerization search space. Artificial intelligence techniques are very versatile and effective stochastic modelling and optimization tools and have recently been revived in many scientific fields including materials science [29-30]. The proposed Intelligent Monte Carlo (IMC) simulation approach is a combination of artificial intelligence with KMC to yield (as problem output) the appropriate reaction conditions in response to predesigned polymeric microstructures (as problem input).

In the current study, chain shuttling polymerization has been chosen as a challenging and intricate polymerization system to test the proposed IMC simulation approach [7, 31-33]. In the process represented in Scheme 1, the growing macromolecular chain is able to shuttle between two catalysts showing a significantly different reactivity vs. ethylene and 1-octene, the two comonomers [5]. The trans-metallation reaction occurs via a chain shuttling agent (CSA). The resulting products, olefinic block copolymers (OBCs), are recently developed representatives of macromolecules with a complex, fine-tuned microstructure composed of blocks of statistical copolymers with two different compositions, one being crystallizable and the other amorphous [34]. 


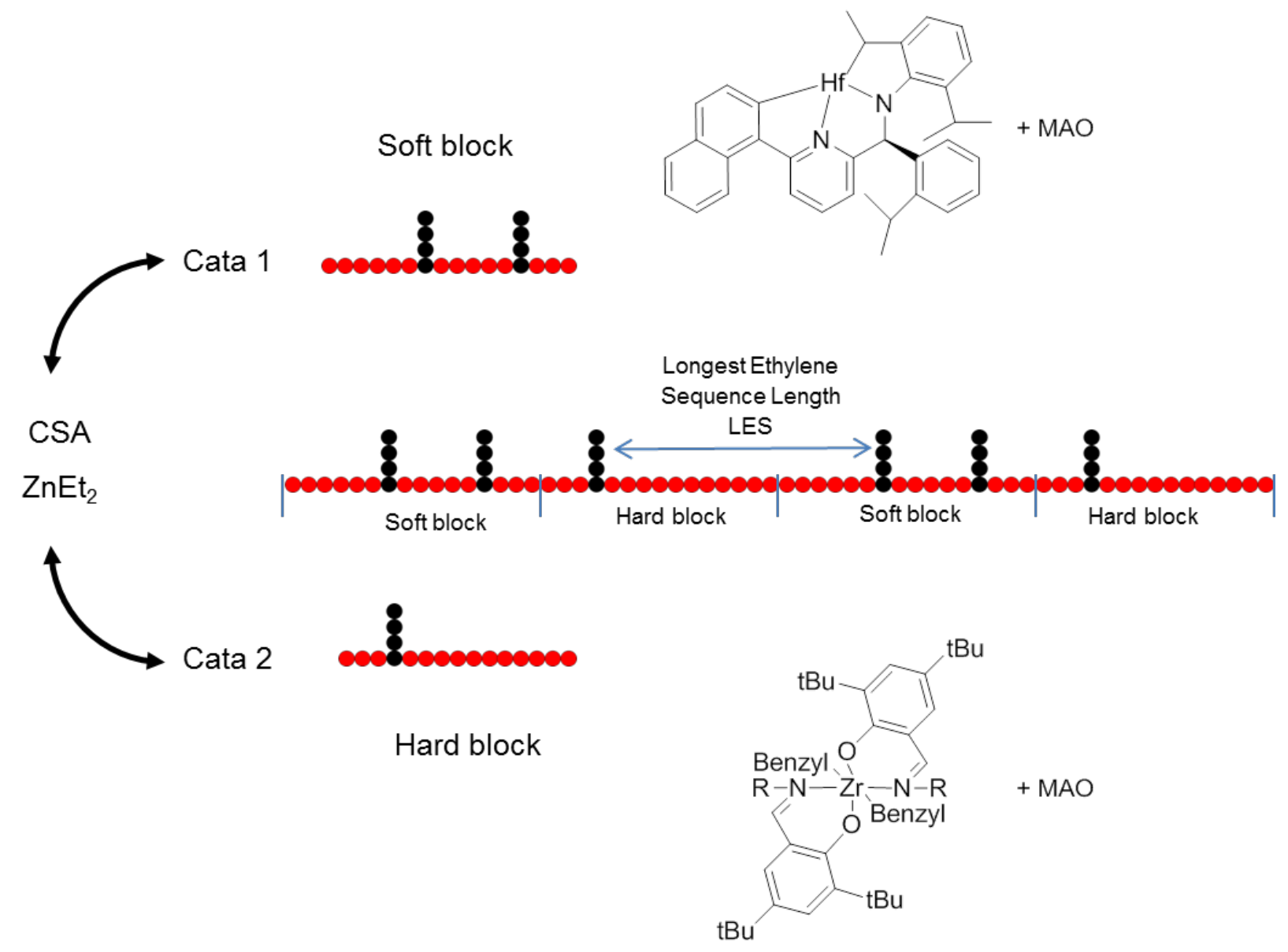

Scheme 1. Ethylene/1-octene chain shuttling polymerization leading to olefinic block copolymers (OBCs) [5]. CSA is the chain shuttling agent and MAO methylaluminoxane. $\mathrm{R}=2$ methylcyclohexyl (cata stands for catalyst).

We previously reported on microstructural changes in the semi-batch chain shuttling polymerization of ethylene and 1-octene using KMC simulation [35-38]. The KMC simulation was able to quantify many signature distributional characteristics of ethylene/1-octene copolymerization, such as the block composition, referred to as $C 8 \%$, the block length represented by the number-average degree of polymerization $\overline{D P}_{n}$, the relative proportion of the blocks described via the hard block content ( $H B \%$ ), the number of linking points between blocks ( $L P$ ), ethylene sequence length ( $E S L$ ), and also the longest ethylene sequence ( $L E S$ ). Although the developed KMC code was capable of precisely simulating the sequence distribution and microstructure of the olefin block copolymer chains (y dependent variables) in a virtual reactor in response to input reaction conditions ( $x$ independent variables), in practical 
applications, the desired properties of the OBCs can be specified in advance, whereas the reaction conditions such as monomer molar fraction, catalyst package composition, temperature, and CSA concentration (that would yield the OBC desirable properties) are not readily evident and they thus have to be determined. As mentioned above, the reverse simulation going from the output macromolecular properties to the input reaction conditions requires random searches of a large multi-variable space for the optimum conditions for the synthesis of OBCs with predesigned microstructures.

The developed IMC approach is able to utilize synergistically the power of KMC and artificial intelligence to map out the output-to-input variable space of chain shuttling reactions in order to find the optimum reaction conditions irrespective of the number of variables in the input and output search spaces. The IMC approach is faster and more efficient than other available computational tools for predicting and optimizing polymerization reaction conditions for a given copolymer architecture and microstructure, and much quicker (while almost equally reliable) than an empirical approach using pilot-scale reactor experimentation that is usually timeconsuming and costly.

\section{MODEL DEVELOPMENT}

KMC simulation is a unique mathematical tool for virtually synthesizing and visualizing complex polymerization reactions. It has been successfully applied to a variety of macromolecular reactions. Despite its remarkable potential, KMC simulation is limited by its inability to 'match' input variables, i.e. copolymerization recipe and operating condition variables, to outputs (e.g. copolymerization chain topological features). To find the optimum reaction recipe/conditions for the synthesis of predesigned chain microstructures, KMC should be equipped with the appropriate optimization tools to intelligently seek, identify, and screen for molecular patterns in the copolymerization search space. As mentioned earlier, artificial intelligence techniques are very versatile and effective stochastic modeling and optimization tools currently employed successfully in many scientific fields [39-41]. Among different computationally intelligent techniques, Artificial Neural Networks (ANNs, biologically-inspired modeling tools) and Genetic Algorithms (GAs, evolutionary optimization methods) have gained much attention in recent years [42-52]. Intelligent Monte Carlo (IMC) simulation is the application of artificial intelligence within 
the KMC framework in order to map the desired chain microstructures to input reaction recipe/conditions. In the following, we describe the IMC approach and demonstrate its use in finding the polymerization conditions warranting the synthesis of predesigned, tailor-made macromolecules.

A two-step modeling and simulation strategy is required for implementing the IMC approach. First, the copolymerization reaction is simulated to virtually synthesize macromolecular chains with complete microstructural details. The first step is accomplished by KMC simulation. In the second step, a multi-objective genetic algorithm is employed to search the copolymerization variable space in an intelligent evolutionary manner for the optimum reaction recipe/operating condition variables. The genetic algorithm generates random copolymerization recipes and essentially recalls the KMC simulator to synthesize and visualize the macromolecular chains for each recipe. By applying this reverse strategy, each copolymerization recipe behaves like a genotype and is precisely related to a set of virtually synthesized macromolecules, which act as phenotypes. The predictions of the KMC simulator are subsequently modeled with artificial neural networks (ANNs) to reduce the frequency of invoking the KMC simulator with long execution times. Hence, the stochastic modeling of the KMC predictions by trained ANNs dramatically reduces the computational time of IMC (examples of typical computational times will be cited later).

\subsection{First Step: KMC simulation and its corresponding ANN-based modeling}

We previously developed a KMC methodology for simulating copolymerization kinetics and microstructure of the virtually synthesized macromolecules within a relatively large simulation volume $[15,18-19,21,25,28,35-38]$. In this work, complex Olefin Block Copolymers (OBCs) produced by chain shuttling coordination copolymerization reactions have been selected to demonstrate the capabilities of the enhanced IMC approach. OBC copolymerization reactions possess diverse compositional, segmental (hard/soft), molecular, and microstructural properties. In principle, the existence of two catalysts with opposite actions in the chain shuttling copolymerization mechanism involving monomer/comonomer molecules in the presence of a chain shuttling agent, necessitates tracking a massive number of growing macromolecules of different types (identities). We previously tested the KMC simulator by analyzing the 
polymerization kinetics and chain microstructure of OBCs including molecular weight distribution (MWD), copolymer composition distribution (CCD), ethylene sequence length ( $E S L$ ), and longest ethylene sequence ( $L E S$ ) distribution patterns along with their constituent soft and hard blocks in terms of reaction factors, including CSA level, catalyst composition, and monomer molar fraction [35-38]. The simulation algorithm stored the instantaneous characteristics of the dynamic last block along with cumulative information of the static blocks on all virtual chains. The KMC simulator allowed the simulation of a statistically large sample size with a computationally cost-effective execution time. The OBC chains in the reaction volume comprised of soft and hard blocks with fixed length and microstructure, known as static blocks that could not be reorganized. On the other hand, the active terminal blocks of the living or dormant chains were allowed to propagate until experiencing a cross-shuttling or termination to yield a dead chain.

The importance of different reaction variables in tailoring the OBC chains has been discussed in a previous publication [37]. The simulation data on chain characteristics of OBCs can be treated in two ways: (i) final properties, which consist of distribution patterns for blocks, chains, and sequences; and (ii) time-dependent properties, which are representative of the evolutionary variations in chain microstructure. In the first step, the KMC simulator was used to virtually synthesize tailored $\mathrm{OBCs}$ by chain-shuttling copolymerization through an intelligent multiobjective optimization process. In the second step, the KMC simulator was recalled by the genetic algorithm to synthesize new OBCs. Since the KMC simulation is computationally time-consuming and frequently recalled and executed, trained ANNs were used to optimize the microstructure of the OBCs for each intelligently generated copolymerization recipe.

To develop robust ANNs capable of predicting the microstructure of OBCs required a reliable initial dataset on OBCs under different copolymerization conditions to train and test the predesigned ANNs. In that regard, different copolymerization recipes were defined and fed into the KMC simulator based on the independent reaction variables (CSA level, catalyst composition, molar ratio of the monomers) [37]. The CSA level (defined as the logarithm of CSA concentration divided by the initial CSA concentration of $0.27 \mathrm{~g} / \mathrm{L}$ ) ranged from -3 to +1 and determined the length and number of soft and hard blocks per OBC chain [35-38]. The molar fractions of the catalyst package and monomer feed, which ranged from 0.2 to 0.8 , determined the weight 
fractions of soft and hard blocks and the molar fractions of the monomers in the OBC chains, respectively. The input variables for ethylene/1-octene copolymerization, taken from our previous KMC work, are shown in Table 1.

Table 1. Variables applied in KMC simulation of chain shuttling polymerization (CSP) [36].

\begin{tabular}{lll}
\hline Parameter & Value & Unit \\
\hline Initial concentration of ethylene & 2.63 & $\mathrm{~mol} \mathrm{~L}^{-1}$ \\
Solvent initial mass & 1000 & $\mathrm{~g}$ \\
Ethylene initial mass & 200 & $\mathrm{~g}$ \\
1-octene initial molar fraction & $0.2,0.4,0.6$, and 0.8 & - \\
Hydrogen initial mass & 0.072 & $\mathrm{~g}$ \\
CSA initial mass & $0.27 \times 10^{\mathrm{x}} ; \mathrm{x} \in\{-3,-2,-1,0$, and 1$\}$ & $\mathrm{g}$ \\
Catalyst metal initial mass & $1.50 \times 10^{-4}$ & $\mathrm{~g}$ \\
Catalyst 1 initial molar fraction & $0.2,0.4,0.6$, and 0.8 & - \\
\hline
\end{tabular}

Accordingly, the characteristics of 80 virtually synthesized OBCs with diverse microstructural features are shown as $Y$ variables in Table 2. According to Table 2, the properties of the virtually synthesized OBCs were very sensitive to the values of the independent variables and changed over a wide range.

Table 2. Microstructural characteristic of the virtual OBCs synthesized by KMC simulator. $X_{1}, X_{2}$, and $\mathrm{X}_{3}$ are 1-octene mole fraction, catalyst composition, and $\log \left(\mathrm{CSA}\right.$ Level), respectively. $\mathrm{Y}_{1}$ and $\mathrm{Y}_{2}$ are average 1-octene content in soft and hard blocks $(\bar{C} 8 \%)$, respectively, $\mathrm{Y}_{3}$ is the hard block percent ( $H B \%), \mathrm{Y}_{4}$ and $\mathrm{Y}_{5}$ represent average ethylene sequence length in soft and hard blocks $(\overline{E S L}), \mathrm{Y}_{6}$ and $\mathrm{Y}_{7}$ represent number average degree of polymerization of the soft and hard blocks $\left(\overline{D P}_{n}\right)$, and $Y_{8}$ and $Y_{9}$ are the average numbers of 1-octene units per soft and hard block $\left(\bar{N}_{C 8}\right)$.

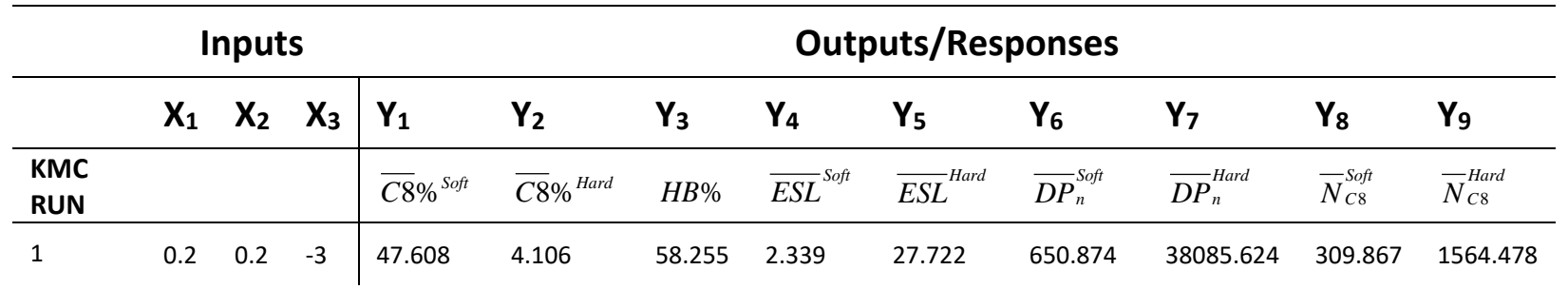




\begin{tabular}{|c|c|c|c|c|c|c|c|c|c|c|c|c|}
\hline 2 & 0.2 & 0.2 & -2 & 47.608 & 4.111 & 58.254 & 2.339 & 27.682 & 591.332 & 12751.269 & 281.528 & 524.735 \\
\hline 3 & 0.2 & 0.2 & -1 & 47.651 & 4.153 & 58.238 & 2.339 & 27.308 & 309.000 & 1669.303 & 147.255 & 69.406 \\
\hline 4 & 0.2 & 0.2 & 0 & 48.002 & 4.579 & 58.083 & 2.339 & 24.030 & 54.613 & 173.840 & 26.219 & 7.969 \\
\hline 5 & 0.2 & 0.2 & 1 & 50.721 & 8.445 & 56.779 & 2.346 & 11.255 & 7.118 & 18.735 & 3.611 & 1.584 \\
\hline 6 & 0.2 & 0.4 & -3 & 46.630 & 3.958 & 34.176 & 2.389 & 28.662 & 722.854 & 32876.143 & 337.071 & 1302.243 \\
\hline 7 & 0.2 & 0.4 & -2 & 46.631 & 3.966 & 34.180 & 2.389 & 28.580 & 667.499 & 7416.399 & 311.278 & 294.411 \\
\hline 8 & 0.2 & 0.4 & -1 & 46.653 & 4.054 & 34.226 & 2.389 & 27.765 & 376.282 & 849.138 & 175.588 & 34.459 \\
\hline 9 & 0.2 & 0.4 & 0 & 46.871 & 4.901 & 34.651 & 2.392 & 21.708 & 71.322 & 87.369 & 33.443 & 4.286 \\
\hline 10 & 0.2 & 0.4 & 1 & 48.532 & 12.006 & 37.816 & 2.426 & 7.344 & 9.076 & 10.040 & 4.407 & 1.206 \\
\hline 11 & 0.2 & 0.6 & -3 & 45.882 & 3.850 & 18.806 & 2.428 & 29.391 & 780.176 & 28914.024 & 357.963 & 1114.093 \\
\hline 12 & 0.2 & 0.6 & -2 & 45.883 & 3.863 & 18.813 & 2.429 & 29.258 & 735.608 & 5218.855 & 337.546 & 201.808 \\
\hline 13 & 0.2 & 0.6 & -1 & 45.893 & 3.991 & 18.881 & 2.429 & 28.010 & 468.605 & 570.842 & 215.150 & 22.805 \\
\hline 14 & 0.2 & 0.6 & 0 & 46.012 & 5.251 & 19.556 & 2.433 & 19.699 & 102.278 & 58.866 & 47.098 & 3.094 \\
\hline 15 & 0.2 & 0.6 & 1 & 46.960 & 15.113 & 24.834 & 2.472 & 5.587 & 12.857 & 7.172 & 6.044 & 1.085 \\
\hline 16 & 0.2 & 0.8 & -3 & 45.261 & 3.763 & 8.013 & 2.463 & 30.007 & 830.011 & 25255.961 & 375.675 & 951.517 \\
\hline 17 & 0.2 & 0.8 & -2 & 45.261 & 3.780 & 8.017 & 2.463 & 29.823 & 804.149 & 4024.560 & 364.007 & 152.286 \\
\hline 18 & 0.2 & 0.8 & -1 & 45.267 & 3.954 & 8.071 & 2.463 & 28.093 & 609.983 & 428.997 & 276.352 & 16.980 \\
\hline 19 & 0.2 & 0.8 & 0 & 45.317 & 5.617 & 8.586 & 2.466 & 17.982 & 180.678 & 44.565 & 82.041 & 2.505 \\
\hline 20 & 0.2 & 0.8 & 1 & 45.750 & 17.900 & 13.003 & 2.492 & 4.587 & 23.727 & 5.734 & 10.884 & 1.027 \\
\hline 21 & 0.4 & 0.2 & -3 & 23.056 & 1.409 & 58.376 & 4.701 & 74.672 & 830.495 & 36636.472 & 191.483 & 516.521 \\
\hline 22 & 0.4 & 0.2 & -2 & 23.059 & 1.411 & 58.371 & 4.701 & 74.424 & 754.144 & 12281.551 & 173.906 & 173.546 \\
\hline 23 & 0.4 & 0.2 & -1 & 23.081 & 1.443 & 58.321 & 4.701 & 71.567 & 394.779 & 1608.190 & 91.139 & 23.244 \\
\hline 24 & 0.4 & 0.2 & 0 & 23.314 & 1.748 & 57.867 & 4.699 & 51.992 & 70.296 & 166.944 & 16.395 & 2.924 \\
\hline 25 & 0.4 & 0.2 & 1 & 24.641 & 4.473 & 54.230 & 4.672 & 14.518 & 9.885 & 17.894 & 2.438 & 0.802 \\
\hline 26 & 0.4 & 0.4 & -3 & 21.790 & 1.320 & 33.908 & 4.967 & 79.492 & 948.639 & 31663.938 & 206.711 & 418.301 \\
\hline 27 & 0.4 & 0.4 & -2 & 21.790 & 1.327 & 33.910 & 4.967 & 78.783 & 875.351 & 7127.176 & 190.760 & 94.696 \\
\hline 28 & 0.4 & 0.4 & -1 & 21.800 & 1.387 & 33.920 & 4.968 & 72.681 & 494.301 & 816.101 & 107.817 & 11.336 \\
\hline 29 & 0.4 & 0.4 & 0 & 21.881 & 1.983 & 34.004 & 4.984 & 41.119 & 94.599 & 83.967 & 20.720 & 1.667 \\
\hline 30 & 0.4 & 0.4 & 1 & 22.001 & 6.852 & 34.387 & 5.149 & 8.428 & 13.101 & 9.535 & 2.888 & 0.654 \\
\hline 31 & 0.4 & 0.6 & -3 & 20.820 & 1.256 & 18.408 & 5.194 & 83.325 & 1049.436 & 27828.497 & 218.494 & 350.158 \\
\hline 32 & 0.4 & 0.6 & -2 & 20.819 & 1.265 & 18.410 & 5.194 & 82.269 & 989.781 & 5046.993 & 206.109 & 63.926 \\
\hline 33 & 0.4 & 0.6 & -1 & 20.821 & 1.354 & 1.354 & 5.196 & 72.642 & 630.248 & 549.010 & 131.373 & 7.447 \\
\hline 34 & 0.4 & 0.6 & 0 & 20.826 & 2.224 & 18.856 & 5.218 & 33.848 & 138.577 & 56.535 & 28.923 & 1.259 \\
\hline 35 & 0.4 & 0.6 & 1 & 20.516 & 8.928 & 21.749 & 5.452 & 6.123 & 18.752 & 6.779 & 3.861 & 0.606 \\
\hline 36 & 0.4 & 0.8 & -3 & 20.016 & 1.205 & 7.728 & 5.400 & 86.761 & 1141.502 & 24482.891 & 228.493 & 295.360 \\
\hline
\end{tabular}




\begin{tabular}{|c|c|c|c|c|c|c|c|c|c|c|c|c|}
\hline 37 & 0.4 & 0.8 & -2 & 20.016 & 1.217 & 7.732 & 5.400 & 85.150 & 1104.721 & 3879.224 & 221.200 & 47.286 \\
\hline 38 & 0.4 & 0.8 & -1 & 20.015 & 1.336 & 7.770 & 5.402 & 71.897 & 837.689 & 412.872 & 168.038 & 5.523 \\
\hline 39 & 0.4 & 0.8 & 0 & 19.999 & 2.469 & 8.133 & 5.419 & 28.696 & 248.662 & 42.759 & 50.000 & 1.057 \\
\hline 40 & 0.4 & 0.8 & 1 & 19.720 & 10.881 & 11.133 & 5.593 & 4.904 & 34.132 & 5.399 & 6.786 & 0.588 \\
\hline 41 & 0.6 & 0.2 & -3 & 10.596 & 0.577 & 52.513 & 9.877 & 176.673 & 1304.194 & 36429.899 & 138.193 & 210.484 \\
\hline 42 & 0.6 & 0.2 & -2 & 10.597 & 0.579 & 52.505 & 9.876 & 175.011 & 1182.713 & 12110.545 & 125.344 & 70.267 \\
\hline 43 & 0.6 & 0.2 & -1 & 10.604 & 0.598 & 52.441 & 9.869 & 159.871 & 619.956 & 1589.107 & 65.764 & 9.532 \\
\hline 44 & 0.6 & 0.2 & 0 & 10.649 & 0.787 & 51.776 & 9.811 & 86.035 & 111.210 & 164.847 & 11.851 & 1.301 \\
\hline 45 & 0.6 & 0.2 & 1 & 10.607 & 2.396 & 46.827 & 9.203 & 16.053 & 16.279 & 17.570 & 1.729 & 0.423 \\
\hline 46 & 0.6 & 0.4 & -3 & 9.409 & 0.515 & 28.068 & 11.101 & 197.450 & 1553.223 & 31462.242 & 146.140 & 162.264 \\
\hline 47 & 0.6 & 0.4 & -2 & 9.409 & 0.519 & 28.068 & 11.100 & 193.268 & 1432.063 & 7051.519 & 134.766 & 36.655 \\
\hline 48 & 0.6 & 0.4 & -1 & 9.404 & 0.555 & 28.048 & 11.102 & 159.573 & 809.251 & 806.544 & 76.193 & 4.486 \\
\hline 49 & 0.6 & 0.4 & 0 & 9.353 & 0.904 & 27.859 & 11.125 & 58.754 & 156.071 & 82.849 & 14.627 & 0.751 \\
\hline 50 & 0.6 & 0.4 & 1 & 8.580 & 3.586 & 26.311 & 11.172 & 8.847 & 22.767 & 9.315 & 1.961 & 0.335 \\
\hline 51 & 0.6 & 0.6 & -3 & 8.513 & 0.471 & 14.264 & 12.260 & 215.231 & 1781.103 & 27252.799 & 151.642 & 128.744 \\
\hline 52 & 0.6 & 0.6 & -2 & 8.513 & 0.476 & 14.266 & 12.260 & 207.813 & 1678.297 & 4963.964 & 142.934 & 23.714 \\
\hline 53 & 0.6 & 0.6 & -1 & 8.506 & 0.527 & 14.278 & 12.267 & 155.077 & 1068.159 & 542.253 & 91.075 & 2.867 \\
\hline 54 & 0.6 & 0.6 & 0 & 8.432 & 1.018 & 14.393 & 12.329 & 44.478 & 236.343 & 55.774 & 20.022 & 0.569 \\
\hline 55 & 0.6 & 0.6 & 1 & 7.593 & 4.598 & 15.104 & 12.796 & 6.314 & 33.574 & 6.592 & 2.569 & 0.304 \\
\hline 56 & 0.6 & 0.8 & -3 & 7.790 & 0.437 & 5.668 & 13.400 & 2.194 & 1993.206 & 24158.214 & 155.280 & 105.936 \\
\hline 57 & 0.6 & 0.8 & -2 & 7.788 & 0.444 & 5.669 & 13.402 & 220.333 & 1931.377 & 3849.548 & 150.539 & 17.127 \\
\hline 58 & 0.6 & 0.8 & -1 & 7.783 & 0.509 & 5.686 & 13.408 & 148.506 & 1463.606 & 408.405 & 114.477 & 2.083 \\
\hline 59 & 0.6 & 0.8 & 0 & 7.732 & 1.129 & 5.850 & 13.463 & 35.711 & 434.938 & 42.163 & 34.035 & 0.477 \\
\hline 60 & 0.6 & 0.8 & 1 & 7.191 & 5.617 & 7.158 & 13.953 & 5.015 & 61.637 & 5.228 & 4.511 & 0.295 \\
\hline 61 & 0.8 & 0.2 & -3 & 3.647 & 0.190 & 41.899 & 27.818 & 524.333 & 2332.332 & 36025.802 & 85.067 & 68.524 \\
\hline 62 & 0.8 & 0.2 & -2 & 3.647 & 0.191 & 41.888 & 27.812 & 508.471 & 2121.120 & 12058.470 & 77.359 & 23.140 \\
\hline 63 & 0.8 & 0.2 & -1 & 3.645 & 0.199 & 41.824 & 27.701 & 397.730 & 1109.221 & 1578.188 & 40.462 & 3.161 \\
\hline 64 & 0.8 & 0.2 & 0 & 3.626 & 0.281 & 41.219 & 26.652 & 125.682 & 198.287 & 163.772 & 7.200 & 0.462 \\
\hline 65 & 0.8 & 0.2 & 1 & 3.408 & 0.942 & 37.483 & 19.003 & 16.867 & 27.151 & 17.388 & 0.927 & 0.165 \\
\hline 66 & 0.8 & 0.4 & -3 & 2.894 & 0.155 & 19.711 & 35.011 & 637.592 & 2865.481 & 31349.508 & 82.932 & 48.793 \\
\hline 67 & 0.8 & 0.4 & -2 & 2.894 & 0.157 & 19.709 & 35.000 & 595.556 & 2640.330 & 7014.604 & 76.433 & 11.028 \\
\hline 68 & 0.8 & 0.4 & -1 & 2.889 & 0.171 & 19.691 & 34.895 & 359.492 & 1492.626 & 802.289 & 43.216 & 1.375 \\
\hline 69 & 0.8 & 0.4 & 0 & 2.847 & 0.307 & 19.522 & 33.837 & 73.250 & 286.182 & 82.331 & 8.176 & 0.254 \\
\hline 70 & 0.8 & 0.4 & 1 & 2.494 & 1.301 & 18.716 & 25.047 & 9.038 & 38.236 & 9.187 & 0.958 & 0.120 \\
\hline 71 & 0.8 & 0.6 & -3 & 2.407 & 0.133 & 9.306 & 42.095 & 738.563 & 3325.084 & 27202.171 & 80.038 & 36.322 \\
\hline
\end{tabular}




\begin{tabular}{llll|lllllllll}
72 & 0.8 & 0.6 & -2 & 2.406 & 0.135 & 9.306 & 42.088 & 658.375 & 3136.358 & 4967.879 & 75.528 & 6.726 \\
73 & 0.8 & 0.6 & -1 & 2.403 & 0.153 & 9.310 & 41.986 & 315.360 & 1996.809 & 539.812 & 48.179 & 0.832 \\
74 & 0.8 & 0.6 & 0 & 2.368 & 0.332 & 9.351 & 40.962 & 51.667 & 438.618 & 55.401 & 10.470 & 0.185 \\
75 & 0.8 & 0.6 & 1 & 2.085 & 1.590 & 9.842 & 32.029 & 6.396 & 57.326 & 6.482 & 1.207 & 0.104 \\
76 & 0.8 & 0.8 & -3 & 2.070 & 0.118 & 3.543 & 48.969 & 825.428 & 3736.867 & 24003.196 & 77.354 & 28.493 \\
77 & 0.8 & 0.8 & -2 & 2.070 & 0.120 & 3.544 & 48.957 & 698.307 & 3618.114 & 3840.034 & 74.973 & 4.642 \\
78 & 0.8 & 0.8 & -1 & 2.068 & 0.143 & 3.551 & 48.882 & 275.143 & 2739.296 & 406.292 & 57.093 & 0.582 \\
79 & 0.8 & 0.8 & 0 & 2.050 & 0.355 & 3.621 & 48.086 & 39.925 & 811.181 & 41.888 & 16.946 & 0.150 \\
80 & 0.8 & 0.8 & 1 & 1.893 & 1.888 & 4.267 & 41.109 & 5.061 & 109.078 & 5.127 & 2.113 & 0.097 \\
\hline
\end{tabular}

Next, a suitable code was developed to establish and train nine separate ANNs for intelligent stochastic modeling of each KMC output parameter of the OBC reaction. In other words, each ANN was responsible for mapping the complex relationship between the input and output variables and predicting one of the KMC outputs. During the training phase, the parameters of the networks, i.e. weights and thresholds (biases), were adjusted to minimize the prediction errors. The error of a particular configuration of the networks was determined by running all training data through the networks and comparing the generated outputs with the desired targets. After completion of the training process, the ANNs had gained the capability to predict the outputs based on any input data similar to the pattern that they had learned. Prior to entering the input data into the ANNs, the following linear transformation (in the range of -1 to +1 ) was used to normalize the input and response variables in order to prevent larger numbers overriding smaller ones, which could lead to premature saturation of hidden nodes.

$$
X_{i}=2 \times\left(\frac{x_{i}-x_{\min }}{x_{\max }-x_{\min }}\right)-1
$$

where $X_{i}$ is the normalized value of $x_{i}$, and $x_{\min }$ and $x_{\max }$ are minimum and maximum values of $x_{\text {, }}$ resspectively. The normalized data were subsequently divided randomly into training and test datasets. $70 \%$ of the data (56 scenarios/recipes among the 80 OBCs produced by KMC) were randomly selected for training the ANNs, while the rest were used for testing the trained ANNs. Five-layer ANNs were defined with 4-3-5-2-1 neurons in the hidden and output layers to model 
the target microstructural features of the $\mathrm{OBC}$ chains with the input layer having three neurons for each independent reaction variable. Figure 1 depicts the architecture of the proposed ANNs.

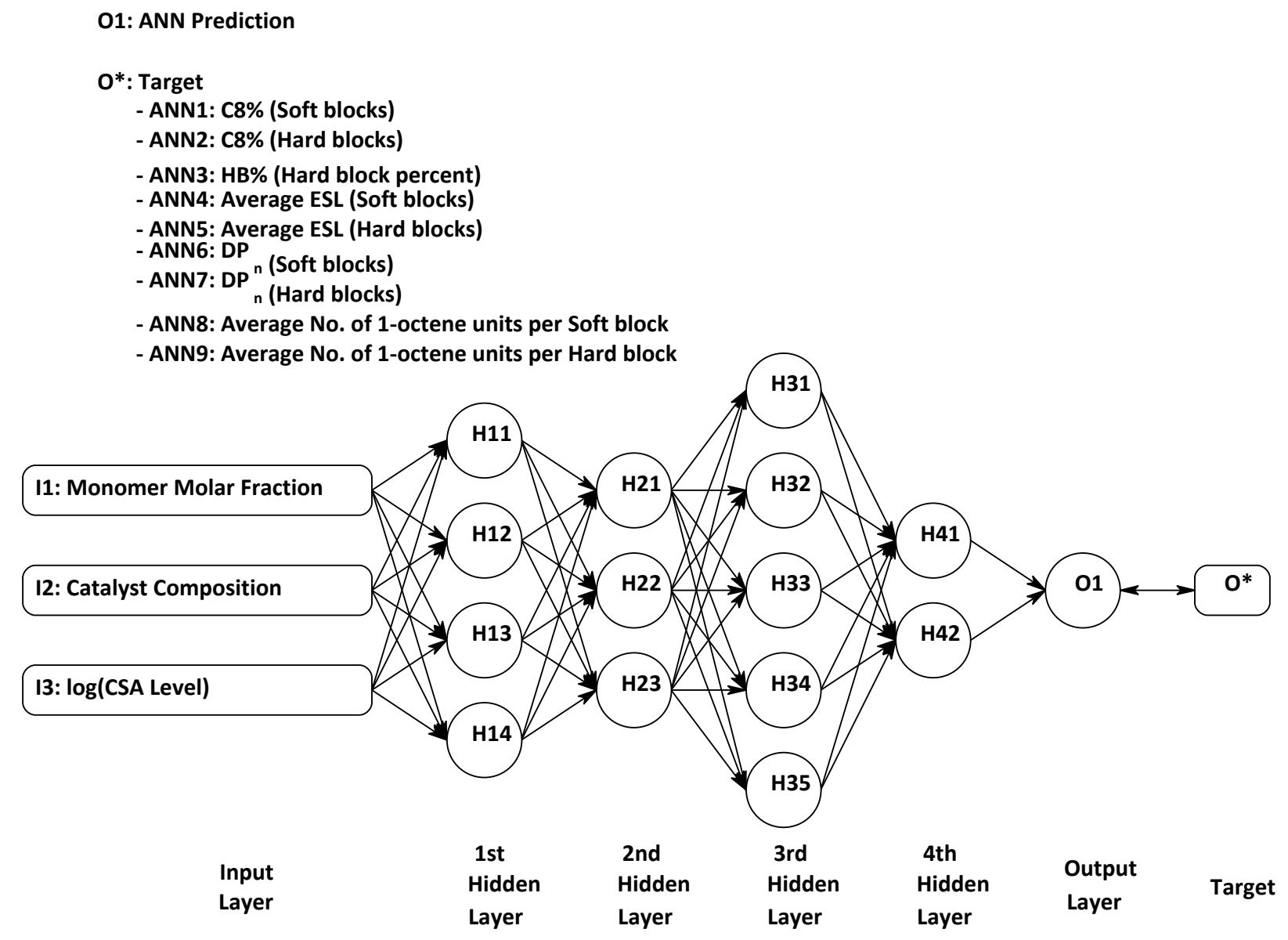

Figure 1. The structure of ANNs used to simulate the microstructure of the OBCs.

The hyperbolic tangent sigmoid function in the hidden and output layers was utilized as the activation transfer function to scale outputs of each neuron within the range of -1 to +1 . The following activation function was used:

$f(x)=\frac{e^{x}-e^{-x}}{e^{x}+e^{-x}}$

where $x$ is the sum of weighted inputs and the bias and $f$ is the hyperbolic tangent sigmoid activation function. A genetic evolutionary algorithm was used to train the ANNs by coding the 
unknown adjustable parameters of the network as chromosomes (Figure 2). Since it is customary to define a bias for a given neuron in the hidden and output layers, the number of unknown biases was equal to the number of neurons. Accordingly, the number of unknown biases and weights were 15 and 51 , respectively.

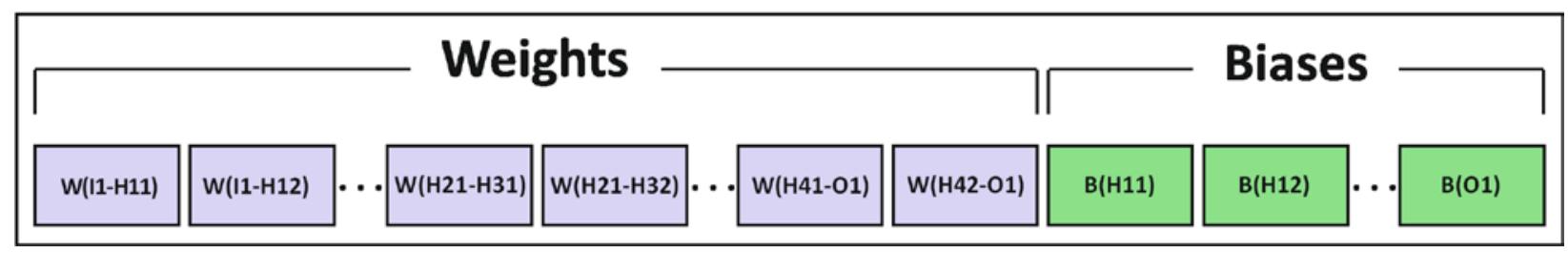

Figure 2. The chromosome structure to codify the adjustable parameters of the proposed ANNs. The weights and biases are represented by $\mathrm{W}$ and $\mathrm{B}$, respectively.

It should be noted that each gene within the chromosome structure could take values in the range of -1 to +1 . An initial population of 50 chromosomes was randomly generated and the ANNs were trained to scan and explore chromosomes by means of the genetic algorithm to find the best chromosome with minimum errors for both training and test datasets. Next, the selected chromosome $j$ was independently entered into the predefined structure of the ANNs for evaluation of the networks based on weights and biases assigned to each chromosome. The normalized input values were separately entered in the network structure to determine the corresponding outputs. Then, the following relationship was used to evaluate the reliability of the ANNs in terms of the mean-squared error (MSE) [53]:

$\operatorname{MSE}(j)=\frac{1}{N_{\text {training }}} \sum_{i=1}^{N_{\text {tarining }}}\left(y_{i, A N N}(j)-y_{i, T \text { arget }}\right)^{2}$

where $N_{\text {training }}$ is the number of data used for training (56 scenarios), and MSE(j) is the MSE for the $j$-th chromosome. Further, $y_{i, A N N(j)}$ is the output of the ANN corresponding to the $i$-th scenario with respect to the $j$-th chromosome, and $y_{i, \text { Target }}$ is the target value for the $i$-th scenario. The training and test errors were related to training and test MSEs as follows: 
Error $=\frac{\sqrt{M S E}}{\operatorname{Max}(\text { Error })} \times 100=\frac{\sqrt{M S E}}{2} \times 100$

where $\operatorname{Max}(E r r o r)$ is the maximum expected error of the networks, which was 2 as the data were normalized between -1 and +1 . After determining the error for each chromosome, the criterion of minimum MSEs was used to sort chromosomes from lowest to highest error to identify the best chromosome. Then, selection, mating, crossover, and mutation operators were applied to produce a new generation of chromosomes. The new chromosome population was entered in the evaluation unit and the optimization process was continued until the selection of optimum chromosomes in accordance with the criterion of minimum MSEs. Merging, sorting, and truncating mechanisms were used for the selection operator, while the roulette wheel mechanism was employed to couple the selected chromosomes. One-point recombination was used to crossover the two parent chromosomes and produce an offspring, and the mutation operator picked randomly one gene from the selected chromosome and stochastically exchanged its value with a new digit in the range of -1 to +1 . The mutation rate was fixed at $20.00 \%$, meaning that the mutation operator was applied to $20 \%$ of the 'child' chromosomes in each iteration or epoch. The values of the parameters used for evolutionary optimization of the ANNs are given in Table 3.

Table 3. The value of the parameters related to single-objective genetic algorithm-based optimization of ANNs.

\begin{tabular}{ll}
\hline Optimization Parameter & Value \\
\hline Initial Population Size & 50 \\
Selection Mechanism & Merge, Sort, and Truncate \\
Mating Mechanism & Roulette wheel selection \\
Crossover Mechanism & Single-point crossover \\
Mutation Rate & $20.00 \%$ \\
Training and Test Errors & $1.00 \%$ and $2.00 \%$, respectively \\
\hline
\end{tabular}

${ }^{a}$ The training and test errors were $3.00 \%$ and $4.00 \%$ in the case of $\mathrm{Y} 3(\mathrm{HB} \%)$, respectively, and $0.50 \%$ in the case of $\mathrm{Y} 7$ and $\mathrm{Y} 9$. 
After completion of the training stage, ANNs generated from the genes of the best chromosomes were evaluated by the test dataset. The optimization process was terminated when a test MSE of less than or equal to a preset MSE was achieved. Otherwise, the optimization algorithm returned to the training stage and the process was repeated.

Results for ANN-based modeling of cumulative 1-octene percent in soft blocks (Y1: $\overline{C 8} \%{ }^{\text {Soft }}$ ) in ethylene/1-octene OBCs are presented in Figure 3. MSE variations of the best chromosomes in the training data showed declining errors as the number of iterations increased. MSE variations for $\overline{C 8} \%{ }^{\text {Soft }}$ in Figure 3 declined from $23.43 \%$ to $0.99 \%$ within 11,229 iterations. The relatively large number of epochs (iterations) and small errors showed that the developed ANNs accurately predicted the variation pattern of $\overline{C 8} \%{ }^{\text {Soft }}$. 

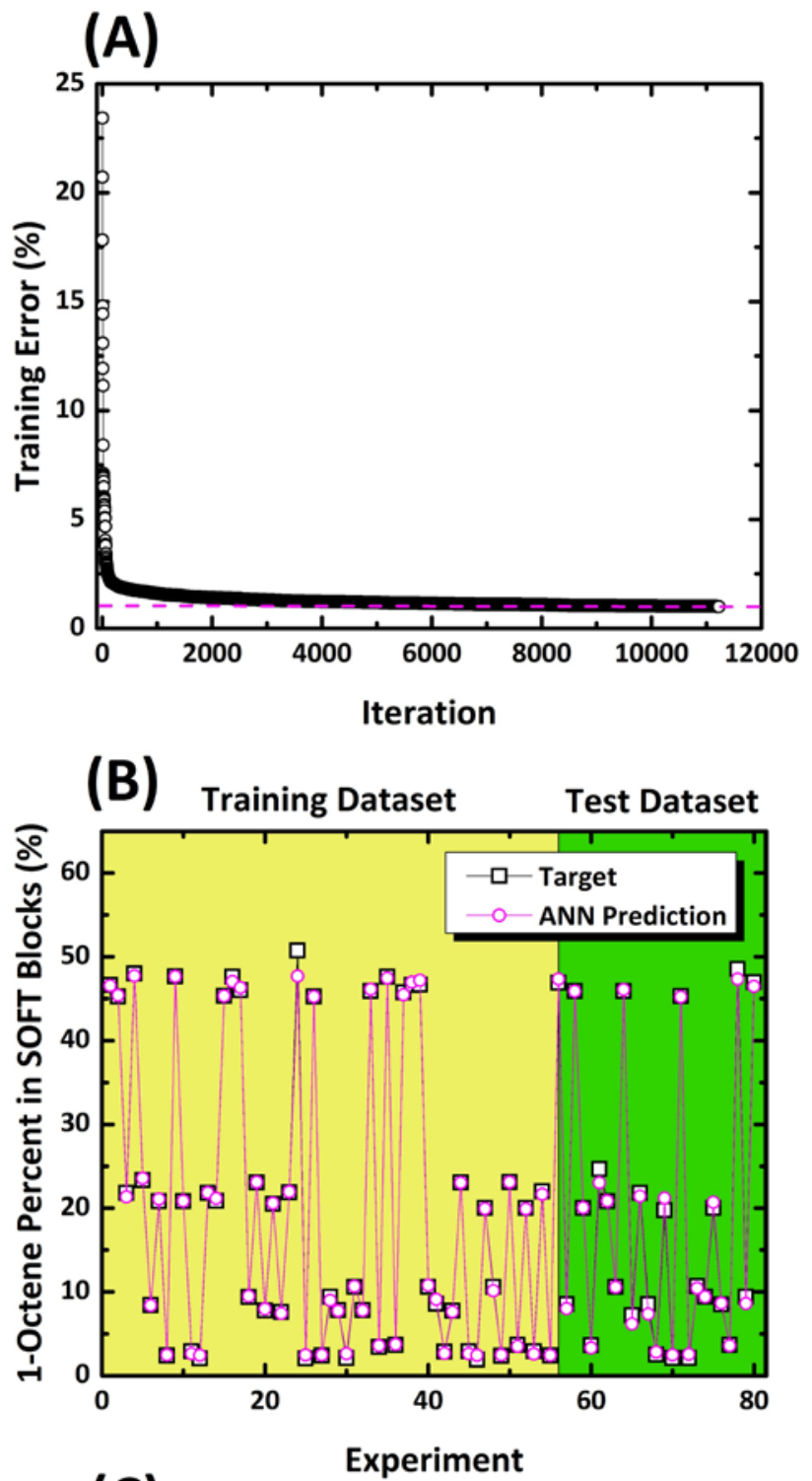

(C)

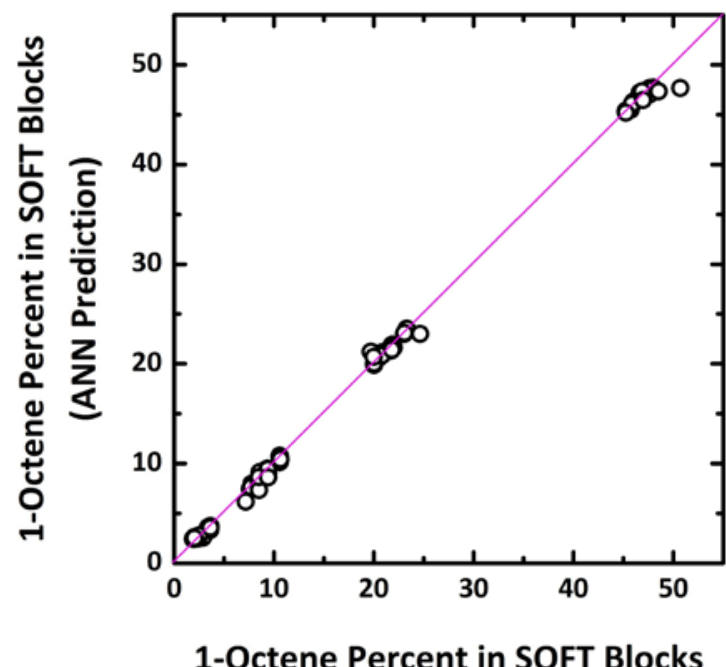

(Target) 
Figure 3. (A) The dependence of ANN training error on number of iterations; (B) Comparison of the KMC outputs with the predicted values based on ANNs corresponding to training (yellow area) and test (green area) datasets for optimization of the response variable $\overline{C 8} \%^{\text {Soft }}$; (C) Correlation between the KMC data and ANN-based predictions for the response variable

$$
\overline{C 8} \%{ }^{\text {Soft }} \text {. }
$$

The performance of the trained ANNs was further tested by comparing the network predictions and the corresponding target values (KMC outputs) for $\overline{C 8} \%{ }^{\text {Soft }}$ for the entire training and testing datasets (Figures $3 \mathrm{~B}$ and $3 \mathrm{C}$ ). The similarity between ANN predictions and target values in Figure 3B is a testament to the accuracy of the developed ANNs in predicting the entire variable space and especially those cases that were not used in the network in the training phase. Further, Figure $3 \mathrm{C}$ shows that the trained ANNs were able to predict $\overline{C 8} \%{ }^{\text {Soft }}$ accurately. Similar results have been obtained for other response variables (Y2 to Y9) and are available upon request. Table 4 lists statistical information about the ANNs for the entire dataset (training and testing), demonstrating the accuracy of the developed ANNs in predicting the microstructural characteristics of OBCs.

Table 4. Results of statistical analyses related to artificial neural networks training and testing.

\begin{tabular}{|c|c|c|c|c|c|c|c|c|c|}
\hline Response & $Y_{1}$ & $Y_{2}$ & $Y_{3}$ & $Y_{4}$ & $Y_{5}$ & $Y_{6}$ & $Y_{7}$ & $Y_{8}$ & $Y_{9}$ \\
\hline & $\overline{C 8} \%{ }^{\text {Soft }}$ & $\overline{C 8} \%{ }^{\text {Hard }}$ & $H B \%$ & $\overline{E S L}^{\text {Soft }}$ & $\overline{E S L}^{\text {Hard }}$ & $\overline{D P}_{n}^{\text {Soft }}$ & $\overline{D P}_{n}^{\text {Hard }}$ & $\bar{N}_{C 8}^{\text {Soft }}$ & $\bar{N}_{C 8}^{\text {Hard }}$ \\
\hline Training MSE & 0.00039 & 0.00039 & 0.00359 & 0.00039 & 0.00099 & 0.00039 & 0.00039 & 0.00039 & 0.00009 \\
\hline Test MSE & 0.00078 & 0.00133 & 0.00575 & 0.00125 & 0.00129 & 0.00109 & 0.00083 & 0.00142 & 0.00034 \\
\hline $\begin{array}{l}\text { Training Error } \\
\text { (\%) }\end{array}$ & 0.99936 & 0.99999 & 2.99926 & 0.99949 & 1.57728 & 0.99987 & 0.99991 & 0.99937 & 0.49875 \\
\hline Test Error (\%) & 1.39431 & 1.82189 & 3.79107 & 1.77011 & 1.79838 & 1.65747 & 1.44154 & 1.88695 & 0.91952 \\
\hline $\begin{array}{l}\text { Max Training } \\
\text { Error (\%) }\end{array}$ & $\begin{array}{l}6.25839 \\
(5)^{\mathrm{a}}\end{array}$ & $\begin{array}{l}6.65651 \\
(20)\end{array}$ & $\begin{array}{l}17.46769 \\
(33)\end{array}$ & $\begin{array}{l}2.11045 \\
(16)\end{array}$ & $\begin{array}{l}8.31046 \\
(56)\end{array}$ & $\begin{array}{l}3.38446 \\
(1)\end{array}$ & $\begin{array}{l}3.87043 \\
(76)\end{array}$ & $\begin{array}{l}5.19647 \\
(16)\end{array}$ & $\begin{array}{l}2.41113 \\
(1)\end{array}$ \\
\hline $\begin{array}{l}\text { Max Test } \\
\text { Error (\%) }\end{array}$ & $\begin{array}{l}3.36469 \\
(25)\end{array}$ & $\begin{array}{l}5.39016 \\
(5)\end{array}$ & $\begin{array}{l}9.66778 \\
(32)\end{array}$ & $\begin{array}{l}4.87684 \\
(80)\end{array}$ & $\begin{array}{l}5.93603 \\
(71)\end{array}$ & $\begin{array}{l}5.33410 \\
(67)\end{array}$ & $\begin{array}{l}3.80190 \\
(19)\end{array}$ & $\begin{array}{l}3.74983 \\
(28)\end{array}$ & $\begin{array}{l}2.71168 \\
(26)\end{array}$ \\
\hline R-Squared & 0.99897 & 0.99523 & 0.98954 & 0.99829 & 0.99531 & 0.99845 & 0.99811 & 0.99778 & 0.99879 \\
\hline $\mathrm{CC}^{\mathrm{b}}$ & 0.99948 & 0.99761 & 0.99476 & 0.99914 & 0.99765 & 0.99923 & 0.99906 & 0.99889 & 0.99939 \\
\hline CoE & 0.99893 & 0.99517 & 0.98932 & 0.99822 & 0.99506 & 0.99843 & 0.99785 & 0.99771 & 0.99870 \\
\hline GoF (\%) & 96.72911 & 93.04744 & 89.66348 & 95.77579 & 92.97125 & 96.03834 & 95.36176 & 95.21012 & 96.39783 \\
\hline CoD & 0.99897 & 0.99523 & 0.98954 & 0.99829 & 0.99531 & 0.99845 & 0.99811 & 0.99778 & 0.99879 \\
\hline
\end{tabular}


a The digit within parentheses specifies the assigned number to the KMC RUN results for maximum error in training or testing MSE. ${ }^{\mathrm{b}}$ CC: Correlation Coefficient; CoE: Coefficient of Efficiency; GoF: Goodness of Fit; CoD: Coefficient of Determination.

According to Table 4, the MSE values of the optimized networks corresponding to training and testing stages were reasonably low. Another beneficial feature of this simulation is the ability to identify the scenario with maximum error in both the training and test phases. It should be noted that 'Scenario' refers to the number assigned to KMC RUN before modeling (as per the number in Table 2), whereas 'Experiment' refers to the assigned number to KMC RUN in the training and testing data sets. It appears that in some cases the large range of variability in the output responses was dependent on the variation pattern of the input reaction factors (as per Table 2). In spite of that, the coefficient of determination corresponding to all studied responses was close to unity.

\subsection{Second Step: Artificial intelligence based multi-objective optimization}

The non-dominated Sorting Genetic Algorithm-II (NSGA-II), a multi-objective version of the conventional GA, was utilized to simultaneously regulate several microstructural features of $\mathrm{OBC}$ chains generated virtually by the KMC simulator [47-48]. NGSA-II stochastically generates structured chromosomes as genotypes, visualizes as well as analyzes the corresponding macromolecules as phenotypes, and subsequently scans and reports the optimized ones that match the predetermined targets. The following approach was used for the artificial intelligencebased optimization of $\mathrm{OBC}$ reactions. First, the three independent variables to be optimized were coded as chromosomes (Figure 4) such that every chromosome had three genes corresponding to the number of independent/input variables. Next, the initial population of chromosomes was generated stochastically to initiate optimization through the evolutionary genetic algorithm. The values of the genes were randomly selected in the normalized range of -1 to +1 (Table 2 ). Then, the fitness of each chromosome (representing a quality indicator of the OBC chains expected from the corresponding scenario) was quantitatively determined by recalling the KMC simulator and the trained ANNs. The desired chromosome was the one satisfying the generation of OBCs with predetermined properties. 


\section{Monomer Catalyst Ratio \\ CSA Level}

Composition

Figure 4. Defined chromosome structure for coding the copolymerization factors.

The principal difference between NSGA-II and conventional GA is in the sorting mechanism. The chromosomes in NSGA-II are sorted based on the concept of domination. Assuming $F_{1}(i), F_{2}(i)$, $F_{3}(i), \ldots$ and $F_{n}(i)$ as fitness values (or objectives) of chromosome $i$, the non-dominated sorting algorithm was used to determine fitness of the selected chromosome with respect to others. It was essential to sort the chromosomes based on order as well as on quality criteria. The former, known as the primary criterion, is of a higher degree of importance in sorting chromosomes. Accordingly, chromosomes were compared to each other to identify and label the dominated chromosome $i$ over $j$ with a Yellow Card based on the following condition:

$i$ dom $j \Leftrightarrow \begin{cases}\forall x: & F_{x}(i) \leq F_{x}(j) \\ \exists y: & F_{y}(i)<F_{y}(j)\end{cases}$

In other words, chromosome $i$ dominated chromosome $j$ if it was not worse than chromosome $j$ for all objectives and strictly better than $\mathrm{j}$ for at least one objective. For each chromosome, the KMC simulator and the previously trained ANNs were recalled to extract fitness functions of each chromosome. After comparing the chromosomes, the non-dominated ones were screened and labeled as the first Pareto front [54-55]. This procedure was repeated to determine the second, third, and $n$-th Pareto fronts. The $n$-th Pareto front was assigned to the chromosomes that had been dominated $(n-1)$ times. After ranking the chromosomes, the secondary criterion known as Crowding Distance, C.D., was implemented for sorting the members of each Pareto front using the following criterion [56]: 
C.D. $(i)=\sum_{x=1}^{N} d_{x}(i)$ where $: \quad d_{x}(i)=\frac{\left|F_{x}(i+1)-F_{x}(i-1)\right|}{\left|\max \left(F_{x}\right)-\min \left(F_{x}\right)\right|}$

where $N$ is the number of objectives, C.D.(i) is the crowding distance of chromosome $i$, and $d_{x}(i)$ is the crowding distance of chromosome $i$ with respect to objective $x$. Applying the domination criterion, all chromosomes were sorted from best to worst with the best chromosomes lying on the first Pareto front (thus satisfying the minimum error in predicting the predefined targets). Next, the selection, mating, crossover, and mutation operators were applied on the sorted population to create a new generation of chromosomes. The adjustable genetic algorithm characteristics are listed in Table 5.

Table 5. Values of algorithm characteristics for multi-objective optimization by NSGA-II.

\begin{tabular}{ll}
\hline Optimization Parameter & Value \\
\hline Initial Population Size & 500 \\
Selection Mechanism & NSGA-II \\
Mating Mechanism & Roulette wheel selection \\
Crossover Mechanism & Single-point crossover \\
Mutation Rate & $15.00 \%$ \\
Number of Iterations & 5000 \\
\hline
\end{tabular}

Using this approach, the KMC simulator benefited from artificial intelligence by hybridization with ANNs and GA. The resulting IMC algorithm is a powerful mathematical tool able to check billions of cases to identify a macromolecular pattern as close as possible to the desired macromolecule. Figure 5 shows the flow chart of the developed IMC algorithm.

The three separate programs based on the computational algorithms and summary flow chart presented in Figure 5 were written in Pascal programming language (Lazarus IDE) and compiled into 64-bits executable FPC 2.6.2. A subroutine based on the Mersenne Twister algorithm was used to generate random numbers for the simulations [57]. The random number generation subroutine satisfied the tests of uniformity and serial correlation with high resolution. The cycle length of the random number generator was $2^{19937}-1$. Simulations were performed on a desktop computer with Intel Core i7-3770K (3.50 GHz), $32 \mathrm{~GB}$ of memory $(2133 \mathrm{MHz})$, under Windows 7 Ultimate 64-bit operating system. The run-time was approximately 10 hours, 85 minutes, and 42 
seconds for each KMC simulation, including ANN-based modeling of each response (both training and test stages), and heuristic optimization of each case study, respectively. In contrast, the training and test stages of nine different ANNs utilized in the current study took around 12.75 hours. Interestingly, the intelligent optimization step recalls the trained ANNs several million times to handle the optimization problem in less than 42 seconds. Hence, as can be observed, the IMC approach is robust and versatile, and at the same time a computationally cost-effective simulation/optimization tool. 


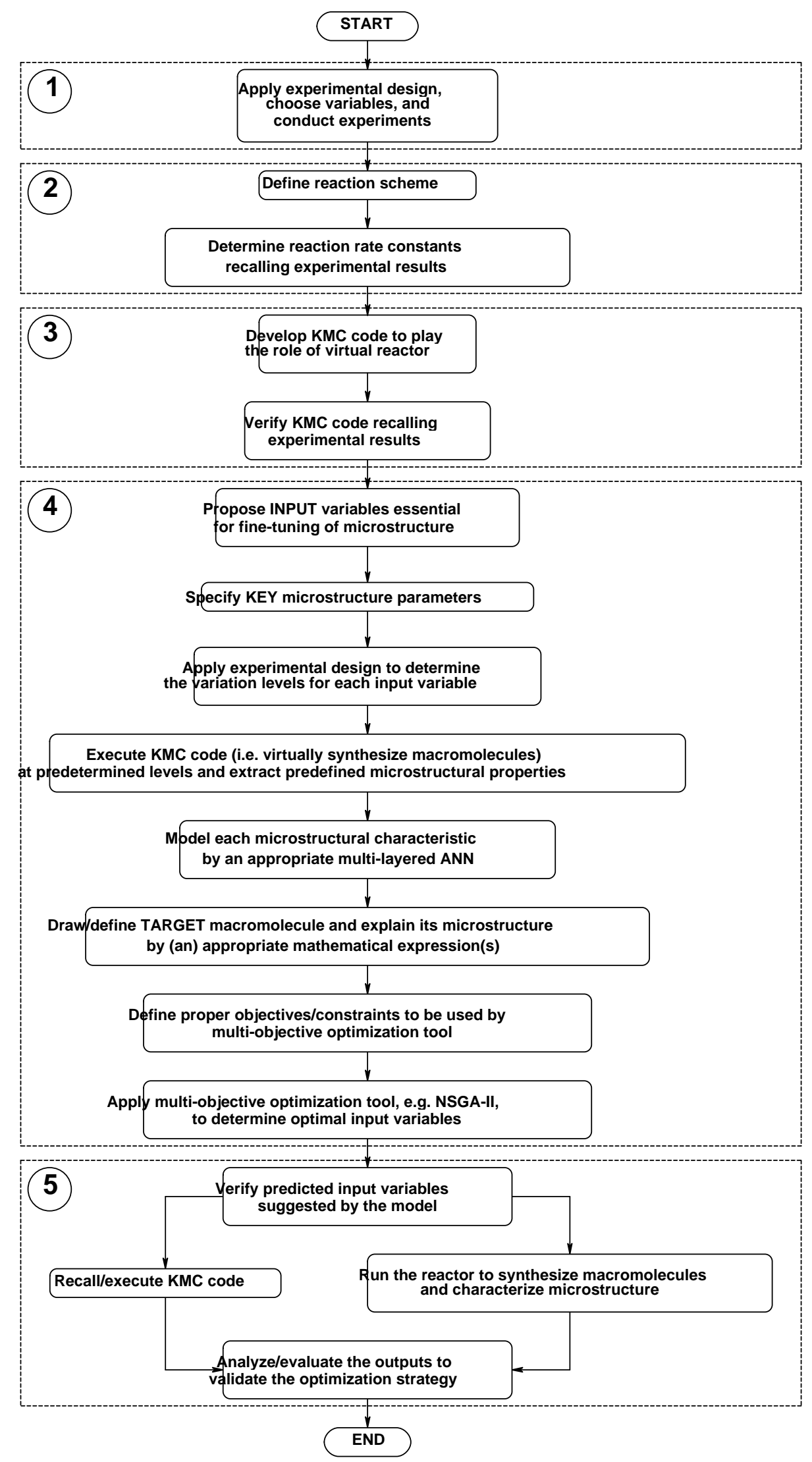

Figure 5. Overall summary flow chart for the IMC simulation approach. 


\section{RESULTS AND DISCUSSION}

Suppose that several microstructural features of the polymer chains in a complex polymerization need to be controlled. This is similar to a situation where one draws on a sheet of paper, as detailed as possible, any hypothetical macromolecule. The IMC simulator acts as an intelligent consultant to bring the virtually drawn ('designed') macromolecule into existence. In this section, the IMC simulator is used to find the reaction conditions for target $\mathrm{OBC}$ chains in a quasi-living ethylene/1-octene copolymerization reaction with specified hard-soft segmental transitions and the corresponding changes in sequence length and sequence length distribution of ethylene units. The target $\mathrm{OBC}$ chains with hypothetically defined molecular characteristics were named $\mathrm{OBC1}, \mathrm{OBC2}, \mathrm{OBC} 3, \mathrm{OBC} 4$, and $\mathrm{OBC} 5$. The selection of a large gene pool, which depended directly on the intervals assigned to the input variables, allowed for an extended search space. Therefore, the variability of genotypes and the corresponding phenotypes determined the predictability range of the IMC simulator. In the following, the virtual synthesis of two tailored OBCs (OBC1 and $\mathrm{OBC2}$ ) using the IMC is presented in detail; for the sake of brevity, the results from the synthesis of $\mathrm{OBC} 3, \mathrm{OBC}$, and $\mathrm{OBC5}$ (which have a greater number of microstructural constraints) are available upon request.

\subsection{OBCs with highly uniform microstructure (OBC1)}

Suppose we are faced with the production of highly uniform OBCs in which 1-octene units are distributed evenly in both soft and hard blocks. The microstructure of a highly uniform OBC is schematically illustrated in Figure 6.

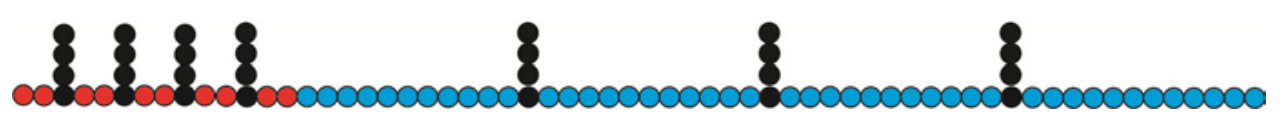

Figure 6. A typical OBC1 macromolecule with uniform distribution of short 1-octene branches (black chains) in soft blocks (red units) and hard blocks (blue units).

In OBC chains with uniform microstructure, the average $E S L$ of the soft blocks $\left(\overline{E S L}^{\text {Soft }}\right)$ is equal to the ratio of the average length of soft blocks $\left(\overline{D P}_{n}^{\text {Soft }}\right)$ to the average number of comonomer 
units per soft block $\left(\bar{N}_{C 8}^{\text {Soft }}\right)$. A similar definition is applied to the hard blocks. In mathematical terms, this requires minimization of the criteria defined by relations (7) and (8):

Objective 1: $\operatorname{MIN}\left|\left(\frac{\overline{D P}_{n}^{\text {Soft }}}{1+\bar{N}_{C 8}^{\text {Soft }}}\right)-\overline{E S L}^{\text {Soft }}\right|$

Objective 2: $\operatorname{MIN}\left|\left(\frac{\overline{D P}_{n}^{\text {Hard }}}{1+\bar{N}_{C 8}^{\text {Hard }}}\right)-\overline{E S L}^{\text {Hard }}\right|$

The first term in parentheses in equation (7) is the average ESL of an ideal uniform soft block having average block length $\overline{D P}_{n}^{\text {Soft }}$ and average number of 1-octene units per soft block of $\bar{N}_{C 8}^{\text {Soft }}$ . The second term in equation (7) is the simulated average ESL of the soft blocks. The virtual synthesis of $\mathrm{OBC} 1$ required concurrent control of six microstructural characteristics of the copolymer chains. Although the IMC simulator was trained and tested with only 80 scenarios, the simulation generated and analyzed billions of macromolecules to find the chain microstructure closest to the target $\mathrm{OBC1}$ with a minimum number of iterations. Figure 7 shows the Pareto front showing the best solutions or the best operating conditions for the synthesis of OBC1.

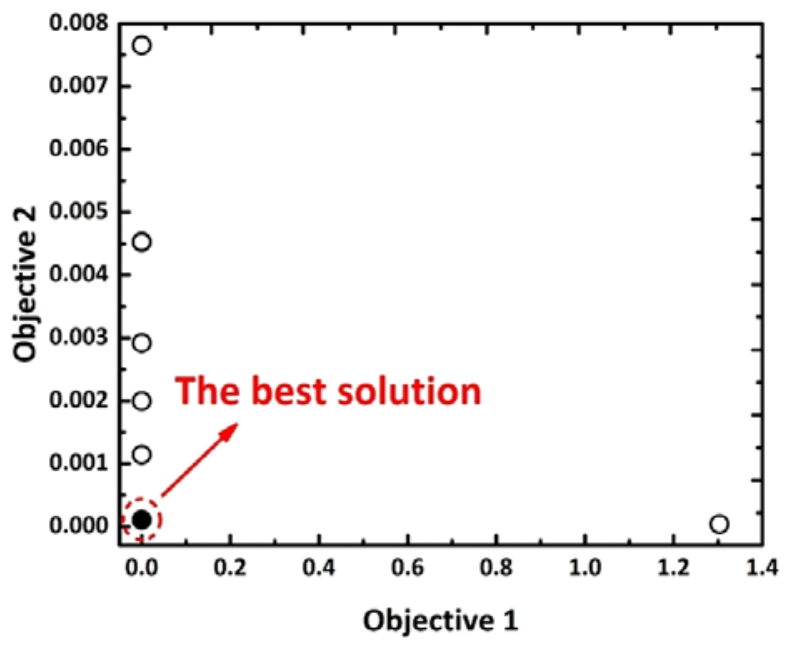

Figure 7. Pareto front for the multi-objective optimization of a highly uniform OBC1. 
Objectives 1 and 2 in the abscissa and ordinate of Figure 7 are the criteria defined by relations (7) and (8), respectively. According to Figure 7, multiple solutions are proposed by the IMC simulator, among which the optimum solution (designated by the red circle) had the lowest values for objectives 1 and 2 with values of $1.88 \times 10^{-4}$ and $1.02 \times 10^{-4}$, respectively. Additional details on Pareto fronts and optimal solutions corresponding to the OBC1 case can become available upon request. The optimal 1-octene molar fraction, catalyst composition, and log(CSA Level) for OBC1 synthesis, as proposed by IMC, are $0.37322,0.65154$, and -0.23192 , respectively, and the corresponding response variables at these optimal conditions are given in Table 6. To validate the authenticity of the IMC results, the optimal reaction conditions were entered into the $\mathrm{KMC}$ simulator to virtually synthesize $\mathrm{OBC}$ chains and determine their microstructural characteristic. The KMC outputs were in good agreement with those of IMC as shown in Table 6 (green row).

Table 6. Optimal input and response variables generated by IMC (highlighted in yellow) and KMC (highlighted in green) simulations for the synthesis of OBC1. Optimal Input Variables

1-octene Molar Fraction: 0.37322

Catalyst Composition: 0.65154

$\log ($ CSA Level) $\quad-0.23192$

Optimal Responses

\begin{tabular}{llllllllll}
\hline & $\overline{C 8} \%^{\text {Soft }}$ & $\overline{C 8} \%^{\text {Hard }}$ & HB\% & $\overline{E S L}^{\text {Soft }}$ & $\overline{E S L}^{\text {Hard }}$ & $\overline{D P}_{n}^{\text {Soft }}$ & $\overline{D P}_{n}^{\text {Hard }}$ & $\bar{N}_{C 8}^{\text {Soft }}$ & $\bar{N}_{C 8}^{\text {Hard }}$ \\
\hline IMC & 29.2706 & 2.06407 & 11.7927 & 4.2255 & 26.9386 & 242.214 & 127.389 & 56.3195 & 3.72889 \\
KMC & 22.9705 & 2.07426 & 15.8438 & 4.74382 & 40.5258 & 227.801 & 88.1609 & 52.4429 & 1.83104 \\
LVa & 1.89253 & 0.11813 & 1.35431 & 2.33881 & 2.19434 & 5.12667 & 7.11763 & 0.92712 & 0.09724 \\
HV & 50.7209 & 17.9001 & 58.3763 & 48.9687 & 825.428 & 38085.6 & 3736.87 & 375.675 & 1564.48 \\
Error (\%) & 12.9026 & 0.05734 & 7.10444 & 1.11157 & 1.65046 & 0.03785 & 1.05177 & 1.03445 & 0.12132 \\
\hline
\end{tabular}

a The lowest value of a given response among 80 scenarios presented in Table $2{ }^{b}$ The Highest value of a given response among 80 scenarios presented in Table 2 .

When the KMC simulator was applied to determine other characteristics of OBC1 chains at optimal conditions (columns not highlighted in Table 6), the results were consistent with those of IMC as shown in Table 6. Figure 8 shows the microstructural characteristics of OBC1 chains; 
this is essentially the copolymer identification card showing both the evolution and final chain distribution of the intelligently synthesized OBCs. We previously found a broad distribution pattern for the average ESL of the OBC chains which made it nearly impossible to evenly distribute 1-octene units along the copolymer chains [35-37]. This was expected as there was only one average LES for each copolymer chain while $\overline{E S L}$ reflected the average length of many ethylene sequences for a given OBC chain. In other words, average LES is determined from the right-hand tail of the ESL distribution curve, whereas the peak of the distribution is the average $E S L$. Figure 8 shows that the ESL distribution curve is relatively narrow even though it was difficult to produce OBCs with similar ESL and LES distributions. The ESL and LES distribution curves for soft and hard blocks in Figure $8 \mathrm{E}$ and $8 \mathrm{~F}$, respectively, show uniform distribution of 1-octene units in hard and soft blocks. Therefore, IMC intelligently maintained the average LES and ESL for soft and hard blocks close to each other to the extent possible, as governed by the underlying polymerization kinetics. 
(A)

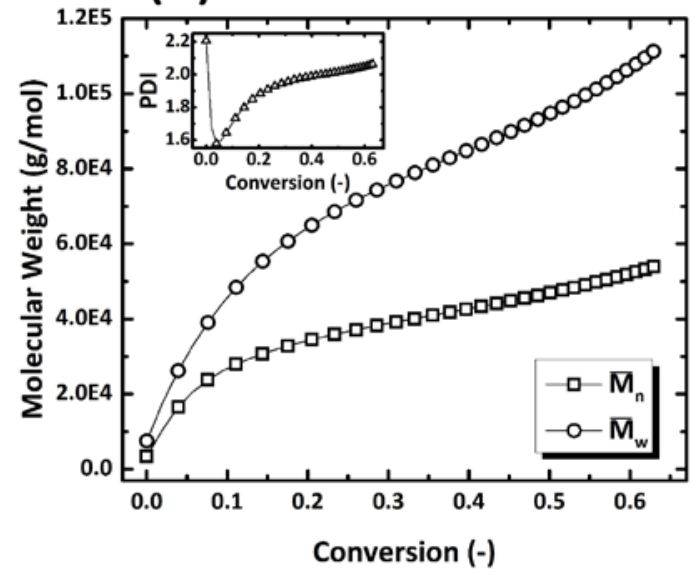

(C)

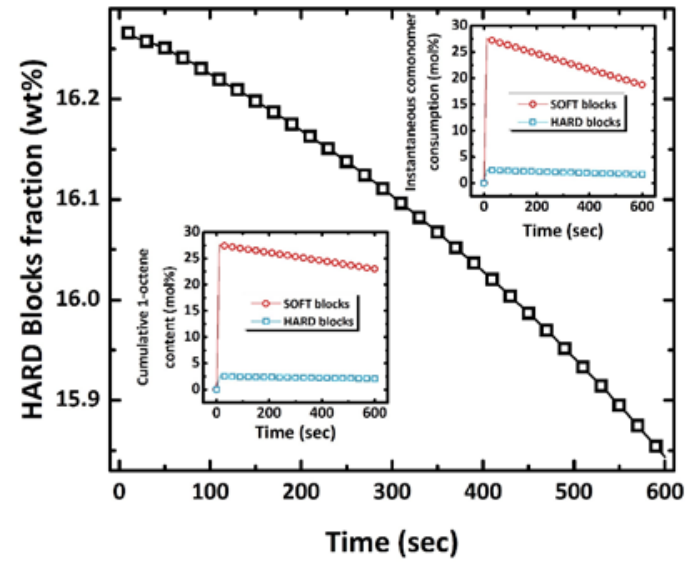

(E)

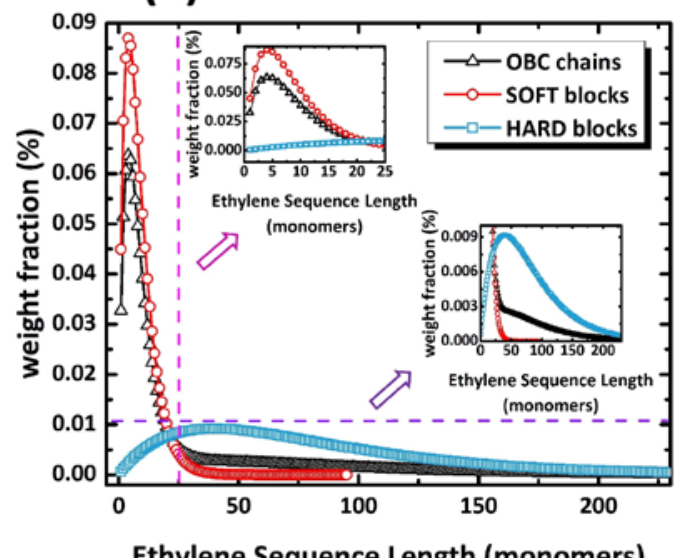

Ethylene Sequence Length (monomers)
(B)

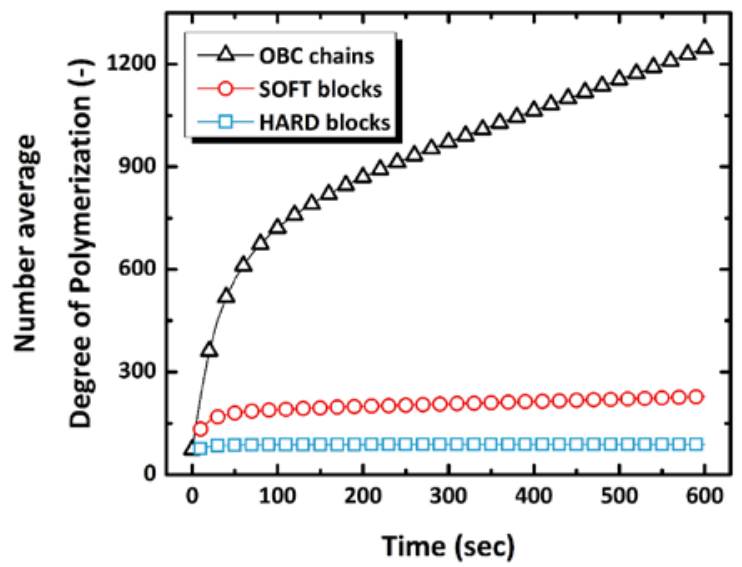

(D)

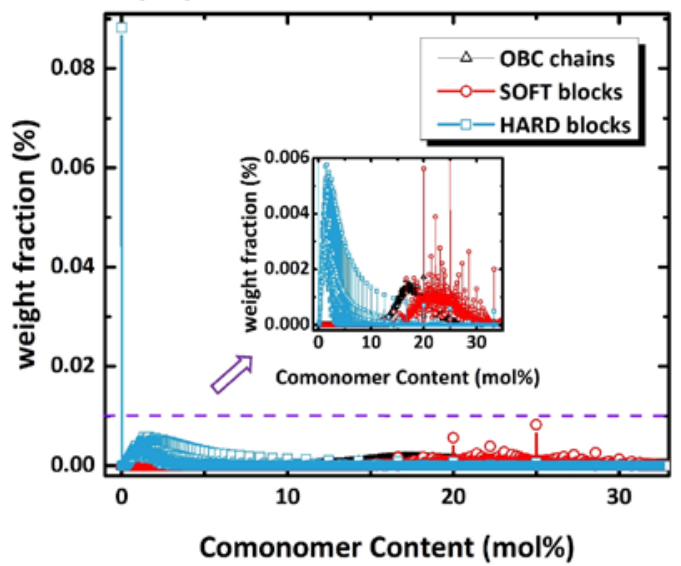

(F)

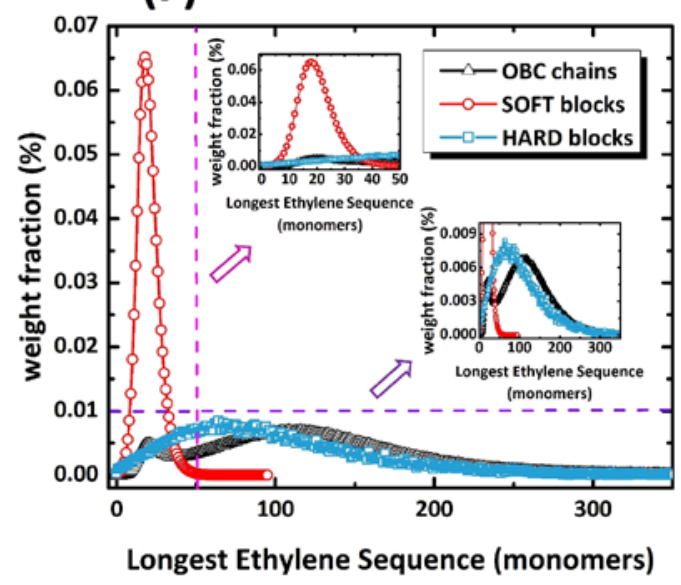

Figure 8. Instantaneous and final characteristics of OBC1.

\subsection{OBCs with highly regular microstructure (OBC2)}


IMC was applied to virtually synthesize highly regular OBC chains in which the comonomer ratio of soft to hard blocks and the ratio of the length of those blocks had predefined values. Those ratios for $\mathrm{OBC} 2$ were 30 and 0.2 , respectively. This was equivalent to synthesizing $\mathrm{OBC}$ chains in which hard blocks were on average 5 times longer than soft blocks; in addition, the comonomer content of the soft blocks was 30 times higher than that of the hard blocks. The microstructure of a highly regular OBC2 is schematically illustrated in Figure 9.

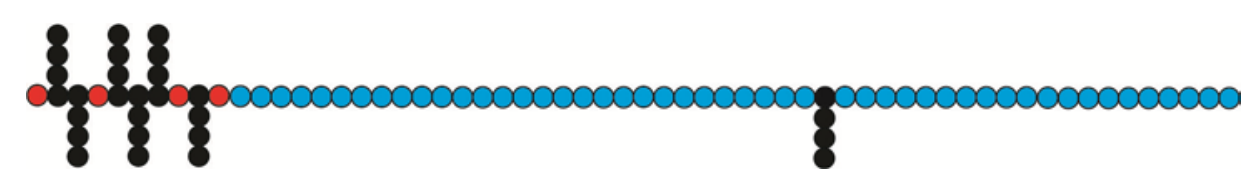

Figure 9. Chain microstructure of a highly regular $\mathrm{OBC} 2$ with red units as the soft block, blue units as a hard block, and black short chain branches as 1-octene comonomer.

From a mathematical standpoint, the following objective functions should be simultaneously minimized to produce the highly regular $\mathrm{OBC2}$ :

Objective 1: $\operatorname{MIN}\left|\left(\frac{C_{8} \%^{\text {Soft }}}{C_{8} \%^{\text {Hard }}}\right)-30\right|$

Objective 2: $\operatorname{MIN}\left|\left(\frac{\overline{D P}_{n}^{\text {Soft }}}{\overline{D P}_{n}^{\text {Hard }}}\right)-0.2\right|$

IMC was applied to simultaneously optimize four molecular characteristics of OBCs. The Pareto front in Figure 10 shows that the errors in synthesizing the target $\mathrm{OBC} 2$ were quite small for the best solutions or the best operating conditions $\left(6.10 \times 10^{-4}\right.$ and $4.95 \times 10^{-4}$, respectively). 


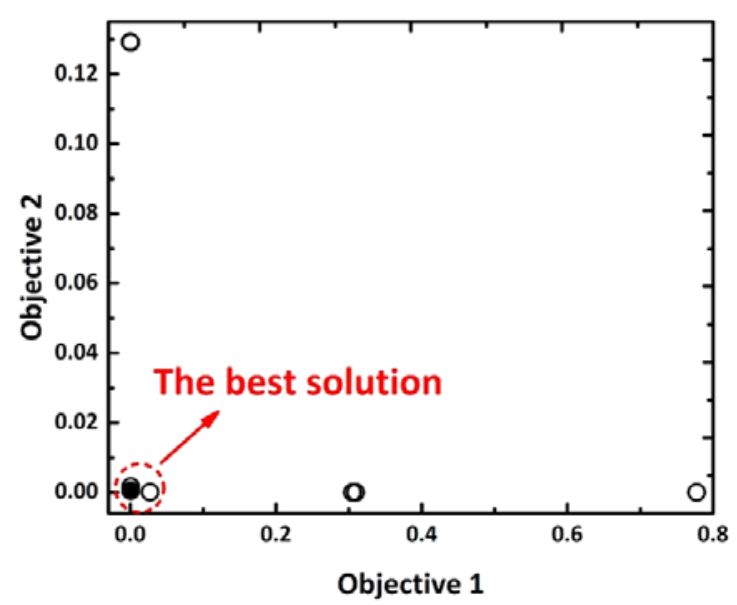

Figure 10. Pareto front for the multi-objective optimization of a highly regular OBC2.

The optimal input variables proposed by IMC for $\mathrm{OBC2}$ synthesis and the corresponding response variables are listed in Table 7.

Table 7. Optimal input variables proposed by IMC for the synthesis of highly regular OBC2 and optimal responses by IMC (highlighted in yellow) and KMC (green).

Optimal Input Variables

1-octene Molar Fraction: $\quad 0.67484$

Catalyst Composition: $\quad 0.20759$

$\log ($ CSA Level): $\quad-1.76063$

Optimal Responses

\begin{tabular}{llllllllll}
\hline & $\overline{C 8}^{\text {Soft }}$ & $\overline{C_{8}}{ }^{\text {Hard }}$ & $H B \%$ & $\overline{E S L}^{\text {Soft }}$ & $\overline{E S L}^{\text {Hard }}$ & $\overline{D P}_{n}^{\text {Soft }}$ & $\overline{D P}_{n}^{\text {Hard }}$ & $\bar{N}_{C 8}^{\text {Soft }}$ & $\bar{N}_{C 8}^{\text {Hard }}$ \\
\hline IMC & 7.90254 & 0.26342 & 48.7163 & 13.3843 & 271.828 & 1493.43 & 7448.71 & 90.7229 & 32.7738 \\
\hline KMC & 7.48723 & 0.40181 & 48.0343 & 13.8085 & 246.898 & 1359.41 & 7629.85 & 101.801 & 30.7512 \\
LV $^{*}$ & 1.89253 & 0.11813 & 1.35431 & 2.33881 & 2.19434 & 5.12667 & 7.11763 & 0.92712 & 0.09724 \\
HV $^{* *}$ & 50.7209 & 17.9001 & 58.3763 & 48.9687 & 825.428 & 38085.6 & 3736.87 & 375.675 & 1564.48 \\
Error (\%) & 0.85056 & 0.77824 & 1.19608 & 0.90975 & 3.02829 & 0.35192 & 4.85654 & 2.95602 & 0.12929 \\
\hline
\end{tabular}

Note: LV and HV as per notes of Table 6.

Figure 11 shows the microstructural characteristics of $\mathrm{OBC} 2$ chains (the copolymer identification card obtained by KMC using optimal polymerization conditions). 
(A)

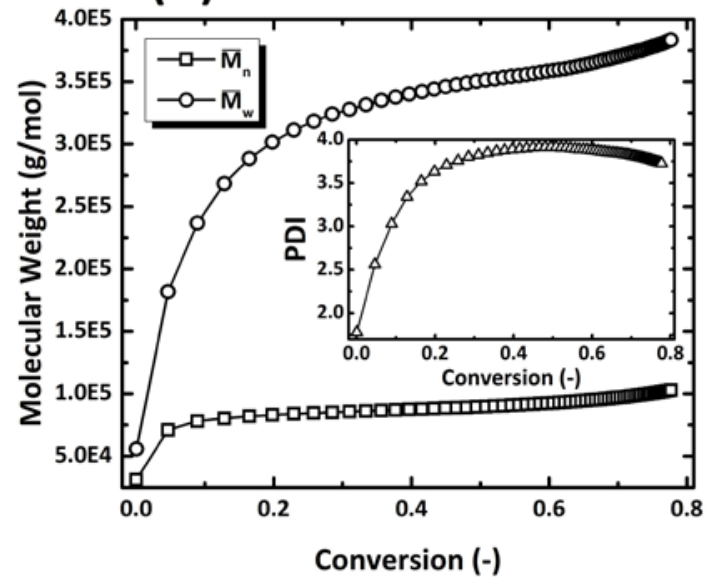

(C)

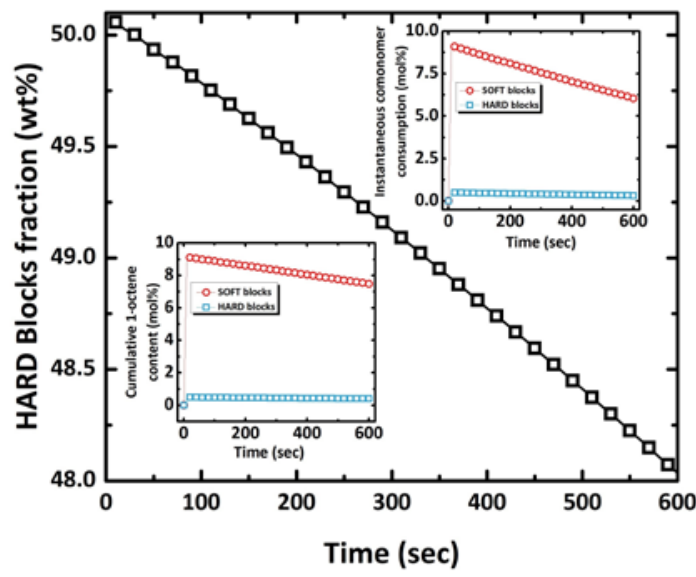

(E)

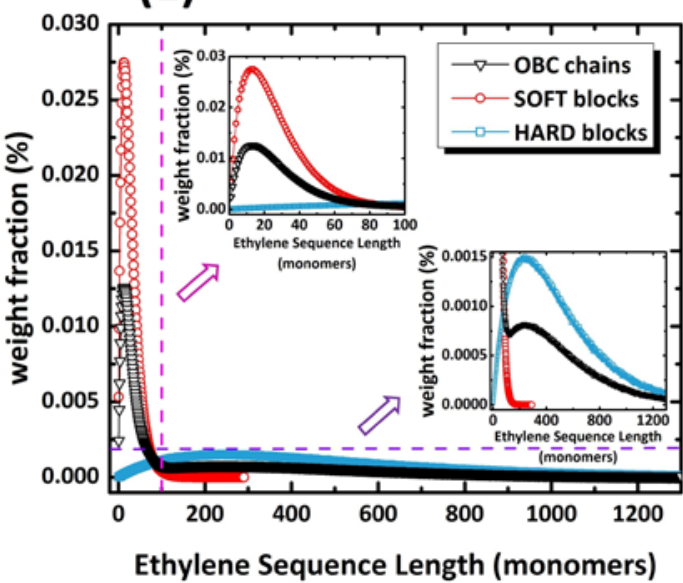

(B)

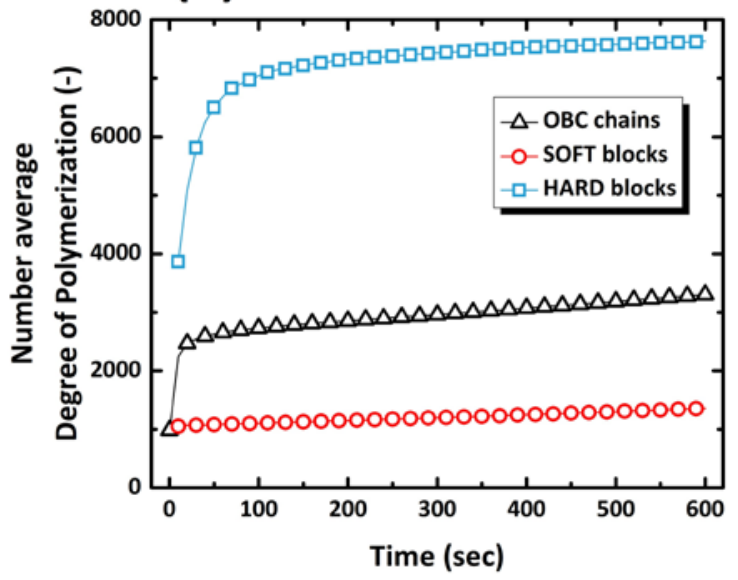

(D)

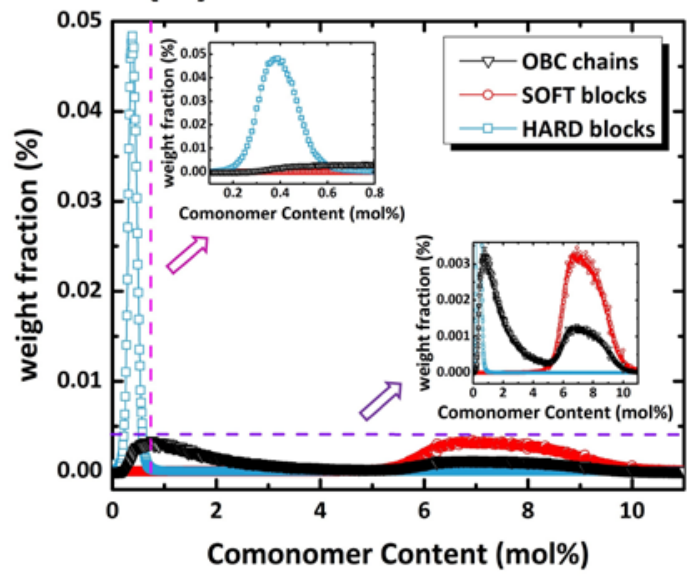

(F)

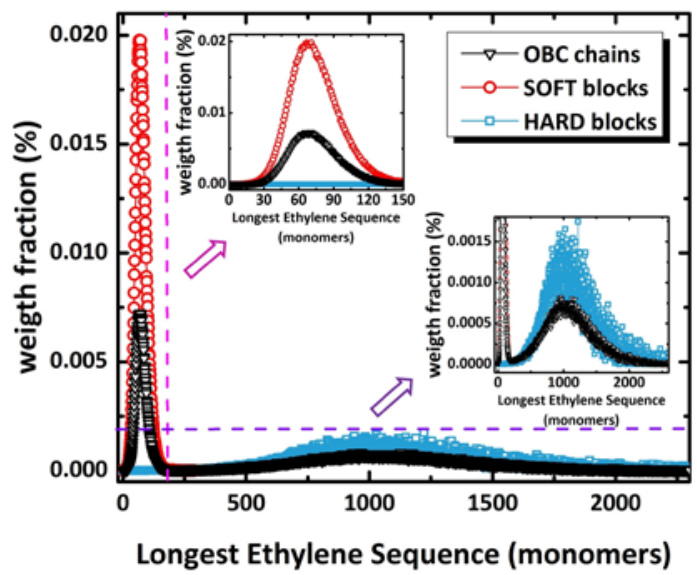

Figure 11. Instantaneous and end-of-batch features of OBC2.

\section{CONCLUSION}


This work proposes a novel approach for simulating the synthesis of polymers with complex microstructures, namely the Intelligent Monte Carlo (IMC) approach. IMC has the ability to make evolutionary decisions to accurately predict the input operating factors in response to target microstructural characteristics of the polymer chains. To illustrate the ability of the IMC approach, the chain shuttling copolymerization of ethylene and 1-octene was selected, considered a rather complex polymerization system. Macromolecular features describing a highly regular and a highly uniform chain microstructure were defined and simulated by the IMC. The power of IMC in concurrently controlling different microstructural characteristics was demonstrated by negligible errors calculated for the objective functions in all cases as compared to those errors obtained for the more tedious (and with longer time-frames) kinetic Monte Carlo (KMC) approach. IMC can thus be viewed as a comprehensive simulation-optimization package. It starts from the simulation of complex polymerizations, learns and identifies the dependency of microstructural features on operating conditions, finds the closest set of operating conditions that lead to control and optimization of several microstructural parameters simultaneously, and checks for the authenticity of the predictions by synthesizing/'designing' the target macromolecule.

\section{ACKNOWLEDGEMENTS}

The Fond Européen de Développement Régional (FEDER) is gratefully acknowledged for funding the France-Wallonie-Vlaanderen Interreg project ELASTOPLAST (PZ).

\section{REFERENCES}

1. W. Guo, D.G. Vlachos, Nature Communications, $2015,6,1$.

2. C.H. Evers, J.A. Luiken, P.G. Bolhuis, W.K. Kegel, Nature, 2016, 534, 364.

3. J.C. Hernández-Ortiz, E. Vivaldo-Lima, M.A. Dubé, A. Penlidis, Macromolecular Therory and Simulations, 2014, 23, 429.

4. J.C. Hernández-Ortiz, E. Vivaldo-Lima, M.A. Dubé, A. Penlidis, Macromolecular Therory and Simulations, 2014, 23, 147.

5. G.W. Coates, R.M. Waymouth, Science, 195, 267, 217.

6. Z. Guan, P. Cotts, E. McCord, S. McLain, Science, 199, 283, 2059. 
7. D.J. Arriola, E.M. Carnahan, P.D. Hustad, R.L. Kuhlman, T.T. Wenzel, Science, 2006, 312, 714.

8. K. Matyjaszewski, Science, 2011, 333, 1104.

9. J.-F. Lutz, M. Ouchi, D.R. Liu, M. Sawamoto, Science, 2013, 341, 1.

10. G. Gody, T. Maschmeyer, P.B. Zetterlund, S. Perrier, Nature Communications, 2013, 4, 1.

11. H. Tobita, Journal of Polymer Science Part B: Polymer Physics, 2011, 39, 391.

12. H. Chaffey-Millar, D. Stewart, M.M. Chakravarty, G. Keller, C. Barner-Kowollik, Macromolecular Therory and Simulations, 2007, 16575.

13. S. Anantawaraskul, P. Somnukguande, J.B. Soares, Macromolecular Symposia, 2012, 312, 167.

14. D. Meimaroglou, C. Kiparissides, Industrial \& Engineering Chemistry Research, 2014, 53, 8963.

15. M. R. Saeb, Y. Mohammadi, H. Rastin, T.S. Kermaniyan, A. Penlidis, Macromolecular Therory and Simulations, 2017, 26, DOI: 10.1002/mats.201700041.

16. P. Van Steenberge, D.R. D’hooge, M.-F. Reyniers, G.B. Marin, Chemical Engineering Science, $2014,110,185$.

17. P. Derboven, D.R. D’hooge, M.-F. Reyniers, G.B. Marin, C. Barner-Kowollik, Macromolecules, $2015,48,492$.

18. M. Najafi, V. Haddadi-Asl, Y. Mohammadi, Journal of Applied Polymer Science, 2007, 106, 4138.

19. M. R. Saeb, Y. Mohammadi, A.S. Pakdel, A. Penlidis, Macromolecular Therory and Simulations, 2016, 25, 369

20. E. Mastan, X. Li, S. Zhu, Progress in Polymer Science, 2015, 45, 71.

21. M.M. Khorasani, M.R. Saeb, Y. Mohammadi, M. Ahmadi, Chemical Engineering Science, 2014, 111, 211.

22. E. Mastan, L. Xi, S. Zhu, Macromolecular Therory and Simulations, 2016, 25, 220.

23. H. Tobita, Macromolecular Therory and Simulations, 2017, 26.

24. P.H. Van Steenberge, D.R. D’hooge, Y. Wang, M. Zhong, M.-F. Reyniers, D. Konkolewicz, K. Matyjaszewski, G.B. Marin, Macromolecules, 2012, 45, 8519.

25. M.R. Saeb, Y. Mohammadi, M. Ahmadi, M.M. Khorasani, F.J. Stadler, Chemical Engineering Journal, 2015, 274, 169.

26. L. Wang, L.J. Broadbelt, Macromolecules, 2010, 43, 2228.

27. R. Szymanski, e-Polymers, 2009, 9, 538. 
28. Y. Mohammadi, M. Najafi, V. Haddadi-Asl, Macromolecular Therory and Simulations, 2005, $14,325$.

29. J. Stajic, R. Stone, G. Chin, B. Wible, Science, 2015, 349, 248.

30. N. Nosengo, Nature, 2016, 533, 22.

31. P. Zinck, Polymer International, 2012, 61, 2.

32. P. Zinck, Polymer International, 2016, 65, 11.

33. Y. Phuphuak, F. Bonnet, G. Stoclet, M. Bria, P. Zinck, Chemical Communications, 2017, 53, 5330.

34. S. Li, R.A. Register, J.D. Weinhold, B.G. Landes, Macromolecules, 2012, 45, 5773.

35. Y. Mohammadi, M. Ahmadi, M.R. Saeb, M.M. Khorasani, P. Yang, F.J. Stadler, Macromolecules, 2014, 47, 4778.

36. M.R. Saeb, M.M. Khorasani, M. Ahmadi, Y. Mohammadi, F.J. Stadler, Polymer, 2015, 76, 245.

37. M. Ahmadi, M.R. Saeb, Y. Mohammadi, M.M. Khorasani, F.J. Stadler, Industrial \& Engineering Chemistry Research, 2015, 54, 8867.

38. M.R. Saeb, Y. Mohammadi, T.S. Kermaniyan, P. Zinck, F.J. Stadler, Polymer, 2017, 16, 55.

39. A. Sfetsos, A.H. Coonick, Solar Energy, 2000, 68, 169.

40. S.A. Kalogirou, Progress in Energy and Combustion Science, 2003, 29, 515.

41. M.D. Fethi, F. Pasiouras, European Journal of Operational Research, 2010, 204, 189.

42. B.G. Sumpter, D.W. Noid, Macromolecular Therory and Simulations, 1994, 3, 363-378.

43. A. d'Anjou, E.J. Torrealdea, J.R. Leiza, J.M. Asua, G. Arzamendi, Macromolecular Therory and Simulations, 2003, 12, 42.

44. R.J. Minari, G.S. Stegmayer, L.M. Gugliotta, O.A. Chiotti, J.R. Vega, Macromolecular Reaction Engineering, 2007, 1, 405.

45. J. Zhang, A.J. Morris, E.B. Martin, C. Kiparissides, Chemical Engineering Journal, 1998, 69, 135.

46. J.C.B. Gonzaga, L.A.C. Meleiro, C. Kiang, R. Maciel, Computers \& Chemical Engineering, 2009, $33,43$.

47. S. Garg, S.K. Gupta, Macromolecular Therory and Simulations, 1999, 8, 46.

48. A. Nayak, S.K. Gupta, Macromolecular Therory and Simulations, 2004, 13, 73.

49. S. Garshasbi, J. Kurnitski, Y. Mohammadi, Applied Energy, 2016, 179, 626. 
50. R. Azari, S. Garshasbi, P. Amini, H. Rashed-Ali, Y. Mohammadi, Energy and Buildings, 2016, 126, 524.

51. B. Baghaei, M. R. Saeb, S. H. Jafari, H. A. Khonakdar, B. Rezaee, V. Goodarzi, Y. Mohammad, Journal of Applied Polymer Science, 2017, 134(33), 45145.

52. M. Hosseinnezhad, M. R. Saeb, S. Garshasbi, Y. Mohammadi, Solar Energy, 2017, 314.

53. S. Koroglu, N. Umurkan, O. Kilic, F. Attar, Simulation Modelling Practice and Theory, 2009, 17, 1267.

54. S.Y. Liong, S.T. Khu, W.T. Chan, Journal of Hydrologic Engineering, 2001, 6, 52-61.

55. X. Blasco, J.M. Herrero, J. Sanchis, M. Martinez, Information Science, 2008, 178, 3908.

56. P.C. Chang, S.H. Chen, C.Y. Fan, C.L. Chan, Applied Mathematics and Computation, 2008, 205, 550.

57. M. Matsumoto, T. Nishimura, ACM Transactions on Modeling and Computer Simulation 1997, $8,3$. 